\title{
Benefits of multidimensional fractionation for the study and characterization of Natural Organic Matter.
}

\author{
Taylor A. Brown ${ }^{\mathrm{ab}}$, Benjamin A. Jackson ${ }^{\mathrm{ac}}$, Benjamin J. Bythell ${ }^{\mathrm{d}}$, Alexandra C. Stenson ${ }^{\mathrm{ae}}$
}

${ }^{a}$ Department of Chemistry, University of South Alabama, 307 N University Blvd, Mobile, AL 36688, United States

btab1006@jagmail.southalabama.edu

cbaj1322@jagmail.southalabama.edu

dbythellb@umsl.edu, Department of Chemistry and Biochemistry, University of Missouri, St. Louis, MO 63121, United States

${ }^{\mathrm{e}}$ Corresponding Author: PHONE: +1 (251) 460-7432, FAX: +1 (251) 460-7359, astenson@southalabama.edu

\begin{abstract}
The belief that chromatographic separation of complex environmental mixtures or natural organic matter (NOM) produces featureless humps from which little, if anything, can be learned is still pervasive. Meanwhile improvements in chromatography and the use of information-rich detection methods have led to meaningful fractionation and revealed consistent data. Here, we build on this work and developed a robust, facile two-dimensional separation with high orthogonality between dimensions. We illustrate that re-injections of fractions (both in the first and in the second dimension) leads to individual peaks at the expected retention times and use information-rich detection to investigate the basis on which NOM is fractionated. We demonstrate unprecedentedly feature-rich chromatograms are observed even with standard UV detection for polar NOM fractions. The second stage of fractionation is demonstrated to separate isomers, providing a direct look at isomeric complexity in NOM as well as a tool to reduce it. Consistent with expectation, but confirmed for the first time through mass spectral data, radicals were detected for NOM components that were generally nonpolar and grouped in the condensed aromatic structure - like region of van Krevelen plots. High-resolution tandem mass spectral data, furthermore, suggests that many higher-MW components of fulvic acids (especially the highly oxidized ones) have formulas that do not match any known compounds in the literature, supporting the hypothesis that fulvic acids are a unique compound-class. Combined, the data illustrate that meaningful reduction in complexity reveals new compositional and structural detail and avails new avenues of investigation.
\end{abstract}

\subsection{KEYWORDS}

Multidimensional HPLC, Separation of Humic Substances, MS/MS of Humic Substances, FTICR MS of Humic Substances, Radicals in Humic Substances 


\subsection{INTRODUCTION}

Complex environmental mixtures from crude oil, tar balls, marine and freshwater dissolved organic matter (DOM) to humic like substances (HULIS) in aerosols and all subgroups (e.g., humic substances (HS), humics acids (HA), fulvic acids (FA)) are a great challenge to analytical chemistry. Here, the umbrella term natural organic matter (NOM) is used to describe all such mixtures. NOM is of environmental, agricultural, industrial, and biomedical importance. For example, NOM, is studied for its effect on limiting nutrients in open oceans[1], its own role as organic nutrient[2,3], carbon cycling/storage[4,5], formation of harmful disinfection byproducts in drinking- and waste-water[6,7], sorption/transport/bioavailability of metals and radioactive waste[8-10], and aerosol composition[11]. Moreover, humics and "humic-mimetics" (synthetically made HULIS) are studied for their antiviral, antibacterial, and cancer-related properties.[12-14] In short, against the backdrop of a rising global population with increasing needs for food/high-yield agricultural soil, safe drinking water, new pharmaceuticals, fuel, clean air and climate normalization, the ability to characterize structure-activity relationship of NOM would constitute an enormous advancement.

Separation is the obvious first step in the study of any complex mixture. Recent reviews which address NOM fractionation include: Nebbioso et al.[15], Minor et al.[16], and Sandron et al.[17]. Early chromatographic results of NOM were not encouraging with the approach characterized by poor recoveries, poor reproducibility, and irreversible retention. Over the last decade, however, the combination of information-rich/selective detectors, improved column technology, and NOM-specific method development, has led to robust fractionation of NOM into physiochemically distinct components, revealing previously unobtainable compositional detail.[15-22] Differences between humic substances from distinct origins and stages of transformation can readily be determined.[18-24] These experiments have shown that fractionation increases the number of detectible constituents. In addition, a positive correlation between polarity and carboxylic content and a negative correlation between polarity, saturation/aliphatic character, and relative size has been established.[18,21-23,25] The general consensus is that early/polar/hydrophilic fractions are highly oxygenated and unsaturated, whereas late/nonpolar/hydrophobic fractions are the opposite.[18,25,26]

To some, a remaining deterring aspect of chromatographic separation of NOM is the absence of features or general appearance of NOM-"humpograms". In the past, some peaks have been induced through the use of two-dimensional-separation and/or information-rich detection.[20,21,27,28] For example, Mawhinney et al.[27] cleverly introduced features by using mass defect information from high-resolution (time-of-flight) mass spectrometry. Similarly, Li et al.[28] obtained striking features with selective protein-like emission/absorbance wavelength pairings in fluorescence detection. Features can also be artificially induced through abrupt mobile phase changes which usually also change the $\mathrm{pH}$ (dissociation decreases in organic solvent), pressure, optical density in UV-Vis detection, and/or ionization potential in electrospray (ESI) sources.[29,30] However, chromatographic separation of NOM is unlikely to ever produce individual peaks for each analyte. To use the chromatography of NOM to its full potential, it is important to abandon the expectation of feature-rich chromatograms, and focus on the true value of separation to the study and characterization of NOM. Prefractionation of NOM 
not only makes most analyses more meaningful but also adds additional information (e.g., polarity, size, electrophoretic mobility, $\mathrm{K}_{\mathrm{ow}}$ ) which enhances interpretability. For example $\mathrm{Li}$ et al. coupled reversed phase chromatography (RP-HPLC) and size-exclusion chromatography (SEC) with multi-excitation/emission scan fluorescence to identify linked fluorescences and improve structural fingerprinting of NOM.[28] Liu et al. combined RP-HPLC with highresolution tandem MS (MS/MS) to see more NOM components and obtain more meaningful MS/MS results. They were able to compare fragmentation patterns for isomers from different NOM samples and link the differences to expected transformations of NOM from swamp to ocean.[21] Gaspar et al. discovered a new structural type (carboxyl-rich alicyclic molecules CRAMs) in NOM based on electrophoretic mobility coupled to high-resolution MS.[22] Woods et al. combined one-dimensional (1D) and two-dimensional (2D) hydrophilic interaction chromatography (HILIC) with NMR and fluorescence to examine correlation between polarity, structural features in NMR, and structural information from parallel factor analysis components (PARFAC). The HILIC separation reduced heterogeneity, decreased interaction with microenvironments and improved the NMR results to the point where individual small acid components could be identified.[31,32] In previous work, we combined RP-HPLC, hydrogen/deuterium exchange (HDX), metal-complexation, low-resolution multistage fragmentation $\left(\mathrm{MS}^{\mathrm{n}}\right)$ and high-resolution MS to obtain structural and compositional information on RP-HPLC fractions (fractions which are further fractionated in the second dimension here).[18] We also demonstrated that fractionating NOM prior to investigating NOM's interaction with other chemicals (i.e., $\mathrm{NaOCl}$ added during water treatment) provides far more definitive results than analyzing bulk NOM.[33] In short, combined chromatographic prefractionation and multiple information-rich analyses have the potential to provide unprecedented structural insight. Structural insight allows more accurate predictions of structure-activity relationships in environmental, pharmacological, toxicological and industrial settings.

As noted above, combining two LC steps has previously been shown to further reduce HS complexity[20,25,29,32] (as well as possibly reduce isomeric complexity[25]), although significant complexity is still observed. Previous work combined SEC with RP-HPLC, RPHPLC with RP-HPLC, or HILIC with HILIC. Unfortunately, none of these combinations provide high orthogonality. Even size and polarity are generally related: charge-exclusion effects in SEC and size-exclusion effects in RP-HPLC decrease orthogonality. Few investigators attempt to optimize or test orthogonality. Among the exceptions are Góra et al. who used wide-pore RPHPLC in the first dimension to avoid size-discrimination effects and narrow-bore SEC in the second step.[29] They were able to show statistically that the separations were not redundant and that injection-to-injection reproducibility was good in the SEC dimension. Here, we improve on such efforts by (1) individually optimizing both dimensions on analytical standards, (2) developing different second dimension gradients for polar and nonpolar first dimension fractions, and (3) evaluating orthogonality of each $2 \mathrm{D}$ pairing. The goal was to obtain multidimensional fractionation methods that were as orthogonal to each other - and the dispersion mechanism within the mass spectrometer - as experimental compatibilities allow.

83 Previous results (e.g., those described above) have shown that the more information-rich the detection, the easier it is to detect physiochemical differences between chromatographic 
fractions. Here, ultrahigh resolution electrospray ionization Fourier transform ion cyclotron resonance MS (ESI-FTMS) was chosen. It has been shown to resolve individual humic ions at the milli-Dalton level and provide molecular formulas [25,34-40] but has some major shortcomings. The fact that FTMS is not quantitative and does not provide detailed structural information is usually overcome by marrying FTMS to other techniques (e.g., NMR, IR, pyrolysis-GCMS, and fluorescence data) and by matching compositional signatures in van Krevelen space to known biomolecules as reviewed by Mopper et al.[41]. The remaining shortcoming that is scarcely addressed in the literature is that even FTMS cannot distinguish between isomers. A major goal here is to specifically tailor multistage chromatographic separation for isomeric fractionation prior to FTMS analysis.

To our knowledge, no other group has focused specifically on the isomeric fractionation of NOM although previous LC-FTMS results have shown ions of identical molecular formula spread over a wide range of retention times $\left(\mathrm{t}_{\mathrm{r}}\right)$, indicative evidence of isomeric separation.[21,22,25,26] Verifying isomeric fractionation is challenging because no technique is capable of analyzing individual analytes without some level of prior separation or differentiation. FTMS comes the closest by distinguishing between analytes so long as they differ in mass.

101

102

103

104

105 MS/MS has long been used to distinguish between isomers for less complex samples. As mentioned above, Liu et al. also used FTMS/MS to distinguish between isomers from different samples.[21] To our knowledge, no group has used MS/MS to examine isomeric separation within a NOM sample. In the past, we have used H/D exchange and recommended it be coupled to MS/MS to provide reliable results.[42] Here, online LCMS/MS is evaluated as a facile and

106 speedy comparison technique. Complimentary HDX analysis, which requires specialized equipment and FTMS analysis to be meaningful, will be examined in future work.

The final impediment to the universal incorporation of chromatography into NOM analysis is that meaningful fractionation of NOM is non-trivial and time-consuming. Although each dimension presented here did require extensive optimization and characterization, the resultant methods are now routinely and reproducibly used by undergraduates after minimal training. Furthermore, neither dimension requires specialized equipment, complex mobile phases, or solvents that are incompatible with HPLC instrument components or sample evaporation to dryness. In short, the proposed methods are facile, robust, and broadly applicable to investigators across the broad swath of NOM-related fields.

\subsection{MATERIALS AND METHODS}

\subsubsection{Sample Designations}

Fractions from the first dimension are identified by "FRC" followed by the first dimension fraction number (e.g., FRC05 and FRC53). Fractions from the second dimension are identified by following the designation of the first dimension by the fraction number in the second dimension. For example, FRC05_11 is the eleventh fraction that resulted from fractionating FRC05 in the second dimension. For both dimensions, fraction numbering started at zero; retention times for relevant fractions are listed in Table 1. 


\subsubsection{Reversed-Phase HPLC Fractionation - First Dimension/ Column 1}

125

126

127

128

129

130

131

132

133

134

135

136

137

138

139

140

141

142

Multidimensional fractionation (MDFrc) was performed offline. Several reviews outline challenges of effective online MDFrc such as: compatibility between mobile phases, compatibility between mobile phase and ESI spray solvent requirements, and flow rate matching to retain resolution in the first dimension during the second (Dugo et al.[43], Francois et al.[44], Malerod et al.[45], and Donato et al.[46]). An additional drawback to online LCMS is that only a limited number of scans can be collected for each "fraction" and additional experiments (e.g., MS/MS or $\mathrm{MS}^{\mathrm{n}}$ ) are largely precluded. In Fourier Transform Ion Cyclotron Resonance mass spectrometers, especially, each MS/MS scan typically takes more than 1 second, severely limiting sampling frequency.

The first dimension of fractionation was conducted as described previously[18,26] with the exception that the injection volume was lowered to $16 \mu \mathrm{L}$, and a different drying procedure was used. Briefly, the method consisted of repeated fractionation of Suwannee River fulvic acid (SRFA, International Humic Substances Society, Standard I) through a Waters X-Bridge Phenyl Column $(4.6 \mathrm{~mm} \times 150 \mathrm{~mm}, 3.5 \mu \mathrm{m}, 130 \AA)$, here referred to as column 1 . The step gradient used is shown in Figure S1. The mobile phase consisted of acetonitrile $(\mathrm{ACN})+0.1 \%$ formic acid and $18 \mathrm{M} \Omega$ deionized (DI) $\mathrm{H}_{2} \mathrm{O}+0.1 \%$ formic acid. The fractions were collected into acid washed test tubes. Each fraction tube was filled with $167 \mu \mathrm{L}(\sim 10 \mathrm{~s}$ of tr) per injection. A more detailed explanation of this procedure can be found in the Supporting Information.

\subsubsection{Reversed-Phase HPLC Fractionation - Second Dimension/Column 2 and 2'}

Choice of Samples: As previously reported[26], early and late column 1 fractions show higher reduction in isobaric complexity than middle fractions. Therefore, an early, highly polar $(\mathrm{FRC05}, \mathrm{tr}=3 \mathrm{~min})$ and late, far less polar $(\mathrm{FRC53}, \mathrm{tr}=11.5 \mathrm{~min})$ fraction were chosen to be refractionated in the second dimension.

Choice of Column: To obtain a second dimension as orthogonal as possible to the first and to the dispersion within the MS, different types of stationary phases were tested (data not shown). Best results were obtained with a Sielc Obelisc N HPLC column $(4.6 \mathrm{~mm} \times 100 \mathrm{~mm}, 5 \mu \mathrm{m}, 100$ $\AA$ ). The Sielc Obelisc N mixed-mode stationary phase consists of cationic head-groups linked by hydrophilic chains to an anionic anchor-group and separates by a complex combination of charge and polarity. Second dimension fractionation of early and late-eluting humic-like standards was optimized separately (Table S1, Figure S1). To avoid cross contamination, separate HPLC columns were used for early and late column 1 fractions, denoted as column 2 and column 2' respectively. The gradients of all three separations are summarized in Figure S1.

Second Dimension Sample Preparation: Samples FRC05 and FRC53 were each removed from the freezer, allowed to reach room temperature, and dissolved in $100 \mu \mathrm{L} 18 \mathrm{M} \Omega \mathrm{DI} \mathrm{H}_{2} \mathrm{O}$ then sonicated and centrifuged for $\sim 5$ minutes. Aliquots $(90 \mu \mathrm{L})$ were transferred to $250 \mu \mathrm{L}$ plastic autosampler vials. The remaining $10 \mu \mathrm{L}$ were stored in a $-80^{\circ} \mathrm{C}$ freezer for mass spectral analysis of the starting material. 
162

163

164

165

166

167

168

169

170

171

172

173

174

175

176

177

178

179

180

181

182

183

184

185

186

187

188

189

190

191

192

193

194

195

196

197

198

199

FRC05 Second Dimension Gradient - Column 2 Method: Early eluting column 1 fractions were separated in the second dimension through a method in which the mobile phase gradient was held isocratic at $100 \%$ aqueous ( $18 \mathrm{M} \Omega \mathrm{DI} \mathrm{H} \mathrm{H}_{2} \mathrm{O}+0.1 \%$ formic acid) for $14 \mathrm{~min}$, then increased to $10 \% \mathrm{ACN}+0.1 \%$ formic acid at 15 minutes, and held at $10 \%$ for 1 min (Figure S1). The column was washed with $90 \% \mathrm{ACN}+0.1 \%$ formic acid from $17 \mathrm{~min}$ to $25 \mathrm{~min}$. Finally, the column was re-equilibrated at $100 \%$ aqueous for the next injection (25-30 min). The flow rate was $1 \mathrm{~mL} / \mathrm{min}$. The injection volume was $30 \mu \mathrm{L}$. Fractions from three injections were pooled by collection into acid washed microcentrifuge tubes numbered FRC05_00 - FRC05_99. Each fraction tube was filled with $380 \mu \mathrm{L}(\sim 23 \mathrm{~s}$ of tr) per injection. After completion of each run, the contents of the microcentrifuge tubes were dried on a Labconco CentriVap and stored at $-80{ }^{\circ} \mathrm{C}$.

FRC53 Second Dimension Gradient - Column 2' Method: The gradient began at 5\% ACN + $0.1 \%$ formic acid (i.e., $95 \% 18 \mathrm{M} \Omega \mathrm{DI} \mathrm{H}_{2} \mathrm{O}+0.1 \%$ formic acid), increased to $25 \%$ at 2 minutes, increased to $90 \%$ at 17 minutes, and decreased to $5 \%$ at 25 minutes. The flow rate was 1 $\mathrm{mL} / \mathrm{min}$. The injection volume was $30 \mu \mathrm{L}$. The fractions were collected into dry, acid washed microcentrifuge tubes numbered FRC53_00 - FRC53_99. Each fraction tube was filled with $380 \mu \mathrm{L}(\sim 23 \mathrm{~s}$ of $\mathrm{tr})$ per injection.

\subsubsection{Offline ESI-FTMS - Second Dimension/Column 2 and 2' Fractions}

Sample Preparation: Samples FRC05_17,_23,_45, and _67 (trs shown in Table 1) were redissolved in $50 \mu \mathrm{L}$ each of reagent grade isopropanol (ISP). Samples FRC53_11,_28, and_67 (trs also shown in Table 1) were re-dissolved in $100 \mu \mathrm{L}$ each of a 70:30 ISP:18 M $\Omega$ DI $\mathrm{H}_{2} \mathrm{O}$. All samples were vortexed for 1 minute, sonicated for 5 minutes, and centrifuged for 3 minutes.

Data Collection: A custom-built 9.4 Tesla ESI-FT mass spectrometer (National High Magnetic Field Laboratory, Tallahassee, FL) was tuned on SRFA and used to analyze the samples in negative ion mode. Flow rate was $1 \mu \mathrm{L} / \mathrm{min}$, needle voltage was $-3300 \mathrm{~V}$, and the tube lens voltage was $-250 \mathrm{~V}$.

Formula Calculation: For each sample, Composer 6 (Sierra Analytics, USA), was used to calibrate spectra and determine molecular formulas. Elements considered were $\mathrm{C}, \mathrm{H}, \mathrm{O}, \mathrm{N}, \mathrm{S}$, and P. Up to two N, one $\mathrm{S}$, and one $\mathrm{P}$ were considered.

\subsubsection{Offline ESI-FTMS - First Dimension/Column 1 Fractions}

The full Infrared Multiphoton Dissociation (IRMPD) event sequence consisted of: Mass selective accumulation and cooling of ions in the 2nd octopole followed by transfer of ions to the cell. Mass selective accumulation and cooling of ions in 2 nd octopole (i.e., by use of a massselective quadrupole) provides the first level of $\mathrm{m} / \mathrm{z}$ discrimination. From this subset of the ions, further mass-to-charge selection was performed in the FT ion cyclotron resonance cell. To enable analysis of single nominal $\mathrm{m} / \mathrm{z}$ (multiple elemental compositions) or a single elemental composition $\left(\mathrm{C}_{\mathrm{c}} \mathrm{H}_{\mathrm{h}} \mathrm{O}_{\mathrm{o}} \mathrm{N}_{\mathrm{n}} \mathrm{S}_{\mathrm{s}}\right.$ ), Stored Waveform Inverse Fourier Transform (SWIFT)[47,48] massselective ion ejection was utilized. This enables separation of elemental compositions that differ only by the difference between one $\mathrm{O}$ atom and $\mathrm{CH}_{4}(0.0364 \mathrm{~m} / \mathrm{z})$. 
Following SWIFT isolation, analyte $\mathrm{m} / \mathrm{z}$ of choice were irradiated with a $\mathrm{CO}_{2}$ laser, excited to higher radius, then detected. A Synrad (Mukilteo, WA) continuous wave $\mathrm{CO}_{2}$ laser $(\lambda$ $=10.6 \mu \mathrm{m})$ mounted off-axis $\left(\sim 3.5^{\circ}\right)[49,50]$ was employed. Because SWIFT isolation over small frequency ranges can result in some energy deposition in the analyte ion of interest and thus affect in-cell position[51] (i.e., ions are no longer in the center of the cell/conventional laser path), the laser beam diameter was expanded to $\sim 9 \mathrm{~mm}$ by means of a Synrad 2.5 -fold beam expander (Mukilteo, WA). Irradiation periods of 1-4 s were utilized to compensate for the lower fluence. Typical (uncorrected) base pressure for the instrument was $\leq 2 \times 10^{-10}$ Torr, measured by a Bayard-Alpert ionization gauge.

\subsubsection{Online LC-ESI-MS/MS - Second Dimension/Columns 2 and 2'}

Early Fraction FRC05: Sample FRC05 was dissolved in $150 \mu \mathrm{L}$ of $18 \mathrm{M} \Omega \mathrm{DI} \mathrm{H}_{2} \mathrm{O}$, sonicated, and centrifuged for $\sim 5$ minutes. The sample was then analyzed through online lowresolution LC-ESI-MS/MS on a LTQ Velos ion trap mass spectrometer (Fischer Scientific, USA) following the early/column 2 method; $\mathrm{m} / \mathrm{z}$ 297, 371, 383, and 453 were monitored. Collision energy was set to $30 \%$ of maximum, isolation width was $1 \mathrm{Da}$, spray voltage was 5.00 $\mathrm{kV}$, spray current was $37 \mu \mathrm{A}$, source heater temperature was $200^{\circ} \mathrm{C}$, multiplier voltages were 1625 and $-1610 \mathrm{~V}$, and the capillary temperature was $300{ }^{\circ} \mathrm{C}$.

Late Fraction FRC53: Column 2' and the late/column 2' method were used for sample FRC53, and the collision energy was lowered to $25 \%$ of maximum; otherwise, treatment of sample FRC53 was identical to that of FRC05.

\subsubsection{Online LC-ESI-MS/MS - Standards}

To investigate the effect of changing mobile phase composition on LC-ESI-MS/MS fragmentation patterns, solutions $(1 \mathrm{mg} / \mathrm{mL}$ ) of standards $4,11,12,13,17,19$, and 23 (Table S1), representing a range of trs for both second dimension separations as well as a range of molecular weights, were prepared in 50:50 reagent grade $\mathrm{ACN}: 18 \mathrm{M} \Omega \mathrm{DI} \mathrm{H}_{2} \mathrm{O}$. Both the early and late (column 2/2') LC-ESI-MS/MS methods (with corresponding columns) were used for each standard. Standards were mixed with the mobile phase stream through a T-shaped union just prior to the ESI source at a flow rate of $500 \mu \mathrm{L} / \mathrm{min}$. The mobile phase flow rate of the mobile phase $(1000 \mu \mathrm{L} / \mathrm{min})$ was also reduced to $500 \mu \mathrm{L} / \mathrm{min}$ through a split prior to the infusion of standard. Collision energy of either 25 or $30 \%$ was used for each standard, Table S2 lists which collision energy was used for each standard.

\subsection{RESULTS AND DISCUSSION}

\subsubsection{Reversed-Phase HPLC Fractionation - Second Dimension/Column 2 and 2'}

Orthogonality: Figure S2 shows the elution order of standards through column 1. Figures S3 and S4 show elution order of standards on column 2/2' with the two different second dimension methods (trs in Table S1). Figure 1 correlates the trs of the standards for both of these columns to the trs for column 1. The correlation coefficient $\left(\mathrm{r}^{2}\right)$ between for the early/column 2 method and 
the column 1 method was 0.38 , indicating poor correlation between the two dimensions of separation, good orthogonality. The correlation coefficient between the late/column 2' method and the column 1 method $\left(\mathrm{r}^{2}=0.0003\right)$ suggests even higher orthogonality.

Reproducibility: As mentioned above, for humics, features in the chromatograms are primarily induced by the gradient and thus often appear deceptively reproducible. Our data are no exception (Figure S5 \& Figure 2 A, B \& D). Therefore, the best indicator of reproducibility is re-injection of fractions. If fractions elute as single peaks at the expected retention times, the actual fractionation (i.e., the separation of NOM into physiochemically distinct fractions) is indeed reproducible. For NOM, such reproducibility is unlikely to be perfect, given the complexity of the mixture and the potential for in-solution reactions and interactions between NOM constituents and between NOM and mobile phase components (e.g., H-bonding, aggregation, deprotonation). Figure 2 A \& B show UV-Vis chromatograms of the SRFA standard following injection onto column 1 with an early (FRC05) and a late eluting (FRC53) fraction highlighted respectively. Subsequent re-injection of these fractions onto column 1 is illustrated in Figures $2 \mathrm{C}$ (FRC05) and 2E (FRC53). Re-injection of the early-eluting fraction (Figure $2 \mathrm{C}$ ) results predominantly in a clean peak (not a humpogram) at the same retention time as previously. Re-injection of the late-eluting fraction produces a more complex chromatogram (Figure $2 \mathrm{C}$ ). The peaks again appear at the expected retention time, but the added complexity leads to greater breadth of features, consistent with high-resolution FTMS data (discussed below and in the literature $[18,26])$ that suggest that late-eluting fractions remain considerably more complex after one round of HPLC fractionation than early fractions.

Figure $2 \mathrm{~F} \& \mathrm{G}$ show UV-Vis chromatograms of FRC 05 and FCR53 following injection onto column 2 and 2' respectively (each with its own method, as described above). In each chromatogram, the fractions reinjected and shown in Figure $2 \mathrm{H} \& \mathrm{I}$ below are highlighted. Figure $2 \mathrm{H} \&$ I show that isolation of material in the second dimension is also reproducible to within tr differences typical for distinct instruments (LC-UV vs. LCMS) with different dead volumes. Notably, combination of two fractions (Figure 2 I) was thought necessary because of extremely small sample size in the second dimension for late-eluting column 1 fractions. Remarkably, two distinct peaks resulted, indicating that fractions separated by only one collection vial ( 23s) in the second dimension exhibit a level of physiochemical distinctness. Although such results are commonplace for simpler mixtures, they are remarkable for fractionated NOM.

Physiochemical distinctness: The chromatograms for both the early and late (column 2 and 2') methods (Figure 2 F \& G) strongly suggest that further separation did occur in the second dimension. Material was retained on the column throughout the $>20$ minute separation for both second dimension methods. Reinjection did not produce the same chromatogram as before (which would have suggested that no actual separation had occurred) but resulted in primarily singly peaks eluting at consistent retention times. That resultant fractions differ from each other physiochemically is also supported by the re-injection results discussed above. Most strikingly, Figure $2 \mathrm{D}$ shows that re-injection peaks of fractions such as 6, 25, and 49 show no chromatographic overlap, illustrating that such fractions are completely chemically distinct and maintain that distinction during dry-down, storage, and redissolution over time. 
Complexity indicators: Internal averaging is responsible for the typical near-Gaussian (or "hump") distribution of NOM in any dispersion experiment. Thus, even without high-resolution FTMS, or any other information-rich detection method, it is apparent that the second dimension fractions of early-eluting column 1 fractions (Figure 2 left) are chemically far less complex than those of late-eluting column 1 fractions (Figure 2 right). Feature-rich UV-vis chromatograms such as Figure $2 \mathrm{~F}$ are unusual for NOM and indicate that internal averaging has been reduced to a point where individual features become apparent in the chromatogram. Critically, the features in Figure $2 \mathrm{~F}$ are entirely unrelated to any gradient changes. In contrast, Figure $2 \mathrm{G}$ is not feature-rich but resembles a typical NOM humpogram, suggesting that internal averaging still obscures any physiochemical differences between individual components. Consistent with these findings, late eluting column 1 fractions are expected to be more isomerically complex based on previously reported chemical formula information.[18,26] As Hertkorn et al. [52] point out, formulas with high $\mathrm{O} / \mathrm{C}$ and low $\mathrm{H} / \mathrm{C}$ ratios (as encountered in early-eluting column 1-subfrc) allow for fewer possible isomers for any given mass than those with mid-range $\mathrm{H} / \mathrm{C}$ and $\mathrm{O} / \mathrm{C}$ ratios (as encountered in late-eluting column 1 fractions).

Room for Future Improvement: Because charge suppression in the ESI source precludes the use of buffers or salts in the solvent for humics (see, for example, Stenson et al.[34] and references therein), mobile phases were unbuffered. As a result, reproducibility of $\operatorname{tr}$ is poor for standards (Figure 1); also, some standards presented as multiple peaks. As expected for an unbuffered solution, standard deviation in tr is generally highest for early eluting standards, those with the highest $\mathrm{COOH}$ to $\mathrm{C}$ ratio. Figure 2D, meanwhile, shows that reproducibility is remarkable - for NOM; fractions produced single peaks at expected retention times. Samples for Figure $2 \mathrm{D}$ were more concentrated than samples for Figures $2 \mathrm{C}$ or $2 \mathrm{E}$. It is possible that inherent, internal buffering of relatively concentrated NOM contributes to decreased variability in retention behavior. However, Figure S5 suggests that for dilute NOM samples, some deviation in features is observed between 2.2-2.7 min. Carefully controlling the mobile phase $\mathrm{pH}$ and ionic strength might therefore improve reproducibility even further. Scale up would allow for buffering the mobile phase by making additional clean up steps (e.g., through solid-phase extraction post fractionation) feasible. Scale-up would also reduce the amount of time samples sit in solution waiting for additional injections to collect into the same test tube, thereby reducing the risk of NOM constituents reacting with each other and/or mobile phase components. Finally, scale-up would improve second-dimension data analysis and allow for more definitive evaluation of reproducibility in that dimension. Here, Figure 2 I indicates surprising reproducibility in the second dimension for such dilute NOM samples by showing separate peaks for fractions only 23 seconds apart in the original second dimension chromatogram (FRC53_27 and FRC53_29). However, not enough material was available to test the long-term reproducibility of entire chromatograms equivalent to Figure S5for the second dimension. In short, given that the fractionation in both dimensions produces interesting new insights (as detailed below), the next greatest improvement to the process would most likely lie in scaling up to semi-preparative or preparative scale in the first dimension.

\subsubsection{Offline ESI-FTMS - Second Dimension/Column 2 and 2' Fractions}



Compositional overlap (i.e., overlap in assignable formulas): Oxygen class distibutions are shown in Figure 3, van Krevelen plots are shown in Figure 4. Plots of the number of formulas belonging to each heteroatom class can be found in the supplemental information (Figure S6). The typical shift of the near-Gaussian peak distribution to higher $\mathrm{m} / \mathrm{z}$ as the number of $\mathrm{O}$ in a class increases can be seen in Figure S7. Striking in all these results is that although early column 1 fractions (blue) are clearly compositionally different from late column 1 fractions (red), as we previously reported[18,26,42], the second dimension of separation only induces slight additional compositional differences. For example, the oxygen class abundance distribution for the early column 2-subfraction (column 2-subfrc) (Figure 3 blue) is shifted to higher oxygen classes vs. the late column 2'-subfrc (Figure 3 red); as expected and previously reported[18,26,42]. Only a slight additional distribution shift to higher oxygen class and a broadening of relative abundances is observed as tr increases for the early column 2-subfrc (Figure 3 light blue to dark blue).

Compositional overlap between column 2-subfrc from the same starting material is also obvious in the Van Krevelen diagrams (Figure 4). Despite clear chemical distinction suggested by the chromatograms and reinjection results (Figure 2), close inspection hints at only subtle differences. For the early eluting column 1 fractions refractionated (Figure 4 blue), more formulas falling into the lipid and condensed aromatic systems (CAS) -like region of the van Krevelen plots (labeled i and iii respectively) gradually appear with increasing retention time in the second dimension. For the late fractions, new formulas in the upper right quadrant stand out for FRC53_11, but are questionable because they appear in only one spectrum. Overall, compositional overlap between fractions is far more apparent than any potential differences. However, compositional overlap does not mean structural overlap.

New detail from the second dimension: Radicals make up a notable portion of late column 2 '-subfrc spectra but were not found in any early column 2-subfrc. Radicals are displayed in a darker color in Figure 4. From that figure, it is apparent that radicals formed primarily for ions in the CAS-like quadrant of the van Krevelen plot (labeled as iii). As Figures 5 and S7 show, the late column 2-subfrc $\mathrm{CHO}$ radicals roughly follow the same distribution pattern as their even electron counterparts. Their low relative abundance and greater tendency to fragment likely hampers detection in more complex, unfractionated samples and may also have hampered their detection in subfractions of FRC05 here. The fact that radicals are only observed in FRC53subfrc, fractions that contain considerable signal in the CAS - like region of the van Krevelen plot (Figure 4) and that their signal is concentrated in the CAS-like region is also consistent with previous findings regarding which type of NOM more readily forms radicals.[53,54](\& references therein) In general, negative radicals in ESI are believed to form from compounds with low reduction potentials and long conjugated $\pi$-systems (e.g., quinones).[54] More specifically, hydroquinones and quinones in CAS are believed to be important functional groups involved in forming NOM radicals (semiquinones).[53] Aromatic and carboxylic functional groups contribute to stabilizing free radicals, but each of these functional groups on its own is an imperfect predictor for radical content.[53] Specific functional group parings appear more relevant. With the method described here, no radicals were detected in highly polar, $\mathrm{COOH}$-rich, early eluting SRFA (FRC05). COOH-poorer [18], CAS-richer, late eluting material (FRC53) did 
produce radicals. The separations presented here thus present a tool to more closely examine the relationship between NOM composition and free radical content/generation. Separation reduces complexity, allowing for clearer distinction between signals in high resolution analysis. The mass spectra analysis presented here can readily be augmented and fine-tuned by combination with electron paramagnetic resonance (EPR), electron spin resonance (ESR), and NMR, availing new avenues of analysis for NOM.

Isomeric Fractionation: Chromatographic results and re-injection data clearly provide evidence of physiochemical differences between fractions. If the fractions were not different from each other, they would interact identically with the stationary phase in the second dimension and elute at the same time. High-resolution FTMS data of the column 2-subfrc meanwhile indicates very little compositional differences between second dimension fractions from the same starting material. Different interactions with the stationary phase (i.e., vastly different trs) suggests different structures; identical molecular formulas from column 2-subfrc to column 2'-subfrc means that although structure is different elemental composition is not. In other words, the second dimension primarily separates isomers - exactly what is needed to enhance high-resolution mass spectral characterization of NOM.

\subsubsection{MS/MS Analysis}

Because the second dimension separation primarily induced isomeric separation, MS/MS analysis was utilized as a possible tool to verify the isomeric differentiation. Tandem mass spectrometry is time-consuming on the FTMS instruments available for this research. In cyclotron resonance instruments, long transients are required. [55] Thus, FTMS/MS can only be applied to a limited number of mass units before the small amount of sample in any given fraction is depleted. The first step here, therefore, was to investigate whether high-resolution MS/MS provided more information than low-resolutions MS/MS on SRFA fractions to make up for the shortcoming of extremely low sampling frequency.

\subsubsection{High-resolution MS/MS - First Dimension/Column 1}

To our knowledge, this is first time highly fractionated NOM has been subjected to FTMS/MS analysis in which individual isobars are isolated (e.g., segments labeled "iso" in Figure S8) before fragmentation. Findings based on these data (Table S3 \& Figure S8) are consistent with what had previously been reported for unfractionated FA through FTMS/MS[56] and through low-resolution ion-trap MS/MS on highly fractionated FA[18]. Isobars with relatively large, positive mass defects (late eluting from column 1) contain fewer heteroatoms (i.e., O) and therefore produce far fewer fragments of the NOM-typical regular losses (i.e., loss of $\mathrm{H}_{2} \mathrm{O}, \mathrm{CO}_{2}$, and combination thereof). In Figure $\mathrm{S} 8$, it is notable that both late-eluting, highmass-defect isobars also show the less common loss of $\mathrm{C}_{2} \mathrm{O}_{3}$. It is not universally true, however, that late-eluting isobars show more or unique neutral losses.

Because neutral losses are either very low mass or a combination of $\mathrm{H}_{2} \mathrm{O}$ and $\mathrm{CO}_{2}$, highresolution MS is hardly, if ever, needed to identify their formulas. Moreover, because no peak in a mass spectrum can, as of yet, be presumed to correspond to a single analyte and because 
402

403

404

405

406

407

408

FTMS/MS is a comparatively slow technique, only glaring and consistent differences can be detected (e.g., fewer losses for late-eluting isobars). Because MS/MS spectra of early-eluting isobars are essentially identical to each other (Figure S8); as are those of late-eluting isobars, high-resolution dispersion of isobars prior to MS/MS is also typically unnecessary, for highly fractionated samples (e.g., those prepared by the first dimension column 1) to detect the only consistent differences we are, as of yet, able to observe. In other words, the differences in fragmentation pattern between large numbers of structural isomers represented by each FTMS peak are unlikely to produce glaring and reproducible differences that require high-resolution MS/MS to identify. If, for example, in the second dimension later-elution isomers favored the loss of $\mathrm{H}_{2} \mathrm{O}$ over $\mathrm{CO}_{2}$, low-resolution MS/MS would be able to detect the pattern far better (because of its higher sampling frequency) than FTMS/MS at the current capabilities for the instruments available to this research team.

Although it was concluded that FTMS/MS was not best suited to evaluating isomeric fractionation, the FTMS/MS data did add some interesting evidence to the ongoing debate [37] over whether humic substances are unique compounds or simply a random assembly of wellknown biomolecules and their derivatives. Attempts to purchase compounds of identical molecular formula to FA to use as standards in method development by this research team have long revealed that few molecules of identical formula to FA exist in the literature, let alone are available for purchase. Of those that are known, most are either unstable or insoluble in water both criteria which rule them out as NOM stand-ins. The number of known substances of identical formula to NOM components is particularly scarce for the earliest eluting SRFA analytes as is partially illustrated by Figure 6, in which the early eluting SRFA ions (blue) fall mainly outside the range of common molecular pre-cursors for NOM. The observation that few of the formulas detected in early-eluting SRFA produced any hits for known compounds in SciFinder was one of the many considerations that had led to the hypothesis that the early-eluting fractions contained older, highly altered (i.e., oxidized) material, whereas the late-eluting fractions were younger, less altered and still comparatively similar to the original biomolecules that produced the FA.[18]

The negative correlation between fragment mass and number of SciFinder hits with identical formula (Figure 7) illustrates how older, highly degraded NOM (blue Figure 7), likely consists of unique compounds that have not yet been characterized or synthesized (no/few SciFinder hits). These data also suggest that the core structures or backbone of such NOM might be comprised of well-known structural units (large number of SciFinder hits). Statistically, the number of possible structural isomers for each given formula increases markedly with mass, especially for compounds comprised of the low-mass $\mathrm{C}, \mathrm{H}$ and $\mathrm{O}$ elements. However, the number of SciFinder hits decreases drastically (causing Figure 7 to be on a log scale) from lowmass fragments to high-mass fragments/precursor ions. In short, the scientific community knows of compounds that are likely similar (or identical to) the core/backbone of SRFA ions (low-mass fragments), but many of the higher-MW components (especially the highly oxidized ones, blue in Figure 7) are completely unknown.

\subsubsection{On-line LC-ESI-MS/ MS - Second Dimension/-Column 2 and 2',}



MS/MS was used to repeatedly break up $m / z 297,371,383$, and 453 throughout the second dimension separation of each column 1 subfraction (FRC05 and FRC53) to investigate if additional evidence for isomeric separation in the second dimension could be derived from differences in fragmentation patterns. The four $\mathrm{m} / \mathrm{z}$ units were chosen as 'precursor peaks' because of their relatively low isobaric complexity and high relative intensity for all subfractions in previously collected offline ESI-FTMS spectra. Details of other precursor peaks considered and how peaks were chosen are discussed in the Supporting Information (Experimental Detail \& Table S4). Unfortunately, no $\mathrm{m} / \mathrm{z}$ unit was ideal for all subfractions. Late samples showed poorer isobaric purity than early fractions, as discussed above.

Figure S9 shows representative MS/MS spectra of column 2-subfrc. For ions of the same nominal mass, differences in fragmentation pattern are mainly in the relative abundance of certain fragments, not in the types of fragments formed. Unfortunately, abundance information is the least precise measure in ESI-MS, especially for low-abundance ions. Therefore, little additional structural information can be derived from the MS/MS spectra at this stage. However, subtle differences between spectra for ions of the same mass are consistent with structural isomers. To assess such differences, losses of $\mathrm{H}_{2} \mathrm{O}(\mathrm{m} / z, 18)$ and $\mathrm{CO}_{2}(\mathrm{~m} / z$ 44) for samples FRC05 and FRC53 are shown in Figures S10 \& S11. These Figures are plotted as the ratio of fragment to 'precursor peak' abundance normalized to the largest ratio observed in each chromatogram. To provide a representative overview, an example of both the best and worst results obtained were selected for Figure 8. Good results show feature-rich changes in the fragmentation pattern (for the same $m / z$ unit at identical instrument conditions) throughout the parts of the chromatogram where sample elutes (e.g., Figure 8 A-C). These changes in fragmentation patterns suggest that analytes separated throughout the gradient with the same nominal mass are breaking up differently and therefore have different structural composition which supports the argument that isomeric separation is occurring. The least promising results show essentially identical fragmentation patterns throughout (e.g., Figure $8 \mathrm{D}$ ), suggesting persistence of internal averaging. It should be noted that the worst obtained results for sample FRC05 (Figure 8 B) are far superior to the worst obtained results for FRC53 (Figure $8 \mathrm{D}$ ). Less internal averaging/better isobaric purity in subfractions of earlier-eluting column 1 fractions (e.g., FRC05) is consistent with the expected lower isobaric complexity of FRC05 compared to FRC53. Late-eluting column 1 fractions, such as FRC 53, have lower $\mathrm{O} / \mathrm{C}$ and higher $\mathrm{H} / \mathrm{C}$ ratios than early-eluting fractions (Figure 6). As mentioned above, formulas with high $\mathrm{O} / \mathrm{C}$ and low $\mathrm{H} / \mathrm{C}$ can form fewer possible isomers for a given mass than those with $\mathrm{H} / \mathrm{C}$ and $\mathrm{O} / \mathrm{C}$ in the mid-range.[52]

\subsubsection{Online LC-ESI-MS/MS with Standards T'ed in post-LC}

To test whether isomeric features in Figure 8 are not merely artifacts of the gradient, standards $4,11,12,13,17,19$, and 23 were continuously injected after the column (i.e., T'ed into the eluent stream immediately prior to the ESI source, Figure S12). Each standard was repeatedly broken up throughout the mobile phase gradients equivalent to SRFA column 2subfrc above. Because the standards were T'ed in just before the ESI source, the normalized ratios of the fragment ions to the remaining parent peak should, in theory, have remained constant throughout the chromatogram. The normalized ratios for colum 2 and 2' LC-ESI- 
MS/MS methods are shown in Figure S13. A representative collection of the best and worst results obtained were selected for Figure 9. Although subtle variations over time are observed in Figure 9 A \& C, the variations appear random and can readily be distinguished from the real results for column 2-subfrc eluents in Figures $8 \mathrm{~A}-\mathrm{C}$. Little distinction is obvious, however, for results such as Figures $8 \mathrm{D}$ and $9 \mathrm{D}$, which means that the worst results for a real sample, can, in some cases, resemble the worst results for the control. The majority of results for SRFA column 2- subfrc (Figure 8) can, however, readily be distinguished from control spectra (Figures 9 and S-11). Thus, changes in the normalized ratio of fractions to the 'precursor peak' over time for FRC05 and FRC53 indicate that isomeric separation has indeed occurred in the second dimension (more so for the early fraction, FRC05, than the late fraction, FRC53, in agreement with expectation).

\subsubsection{Online LC-ESI-MS/MS - Inferences}

LCMS/MS can be used to evaluate decreases in isobaric complexity. Here, it is obvious from the results that column 2-subfrc still contain multiple isomers at each mass because internal averaging persists as evidenced by broad features (Figure 8). It is also obvious that column 2subfrc from late-eluting column 1 material (FRC 53) exhibit a far greater extent of internal averaging that those from early-eluting column 1 material (FRC 05). In general, fractions can be assumed to be isomerically impure until results from additional fractionation steps result in feature-rich chromatograms that remain quantitatively and qualitatively identical in MS/MS pattern to those in the previous fractionation steps.

\subsubsection{Conclusions}

The data suggest that isobaric complexity is significantly reduced in the first dimension whereas in the second dimension, additional separation is primarily between isomers. Isomeric separation is particularly valuable for mass spectral characterization. Consistent with expectation, but supported for the first time through mass spectral data, radicals were detected for NOM components that were generally nonpolar and grouped in the CAS- like region of van Krevelen plots. High-resolution tandem mass spectral data, furthermore, suggest that many higher-MW components of fulvic acids (especially the highly oxidized ones) have formulas that do not match any known compounds in the literature, supporting the hypothesis that fulvic acids are a unique compound-class. Finally, LCMS/MS provides an evaluation of decreases in isobaric complexity. Combined the data illustrate that meaningful reduction in complexity reveals new compositional and structural detail and avails new avenues of investigation.

\subsection{SUPPORTING INFORMATION}

Details on the collection of fractions FRC00-FRC99 through column 1, details of parent peaks considered and how parent peaks were chosen for online low resolution LC-ESI-MS/MS, and any applicable figures (i.e. Figure S1-12) or tables (i.e. Table S1-4) are shown in the Supporting Information. This material is available free of charge via the Internet at http://pubs.acs.org. 


\subsection{ACKNOWLEDGMENTS}

The authors thank Codey Henderson, Nicole Novotny, Bradley Harris and Jimmie McGehee (undergraduates at the University of South Alabama at the time) for assistance with SRFA fraction collection and method development; Hend Altajjar, Shahrzad Badri and Meghan Letard (undergraduates at the University of South Alabama at the time) for proofreading the manuscript; Drs. Alan Marshall and Amy McKenna for institutional support and access to the 9.4 T FT mass spectrometer; and Logan Krajewski (graduate student at Florida State University at the time) for assistance with data collection using the 9.4 T FT mass spectrometer.

Funding: This work was supported by the National Science Foundation [grant numbers EAR0848635, DMR 1157490, and CHE 1039944]; the State of Florida; and the University of South Alabama.

\subsection{REFERENCES}

[1] H. Takata, K. Kuma, S. Iwade, Y. Yamajyoh, A. Yamaguchi, S. Takagi, et al., Spatial variability of iron in the surface water of the northwestern North Pacific Ocean, Mar.

[2] D.A. Bronk, P.M. Glibert, Application of a $15 \mathrm{~N}$ tracer method to the study of dissolved organic nitrogen uptake during spring and summer in Chesapeake Bay, Mar. Biol. 115

[3] T. Tulonen, K. Salonen, L. Arvola, Effects of different molecular weight fractions of dissolved organic matter on the growth of bacteria, algae and protozoa from a highly humic lake, Hydrobiologia. 229 (1992) 239-252. doi:10.1007/BF00007003.

[4] T. Reemtsma, A. These, M. Linscheid, J. Leenheer, A. Spitzy, Molecular and Structural Characterization of Dissolved Organic Matter from the Deep Ocean by FTICR-MS, Including Hydrophilic Nitrogenous Organic Molecules, Environ. Sci. Technol. 42 (2008) 1430-1437. doi:10.1021/es7021413.

[5] W.C. Hockaday, A.M. Grannas, S. Kim, P.G. Hatcher, The transformation and mobility of charcoal in a fire-impacted watershed, Geochim. Cosmochim. Acta. 71 (2007) 34323445. doi:10.1016/j.gca.2007.02.023.

[6] A. Matilainen, E.T. Gjessing, T. Lahtinen, L. Hed, A. Bhatnagar, M. Sillanpää, An overview of the methods used in the characterization of natural organic matter (NOM) in relation to drinking water treatment, Chemosphere. 83 (2011) 1431-1442. doi:10.1016/j.chemosphere.2011.01.018. 
[7] E.E. Lavonen, M. Gonsior, L.J. Tranvik, P. Schmitt-Kopplin, S.J. Köhler, Selective Chlorination of Natural Organic Matter: Identification of Previously Unknown Disinfection Byproducts, Environ. Sci. Technol. 47 (2013) 2264-2271. doi:10.1021/es304669p.

[8] R. Kautenburger, H.P. Beck, Waste Disposal in Clay Formations: Influence of Humic

[9] K. Wrobel, B.B.M. Sadi, K. Wrobel, J.R. Castillo, J.A. Caruso, Effect of Metal Ions on the Molecular Weight Distribution of Humic Substances Derived from Municipal Compost: Ultrafiltration and Size Exclusion Chromatography with Spectrophotometric and Inductively Coupled Plasma-MS Detection, Anal. Chem. 75 (2003) 761-767. doi:10.1021/ac0261193.

[10] A.R.S. Ross, M.G. Ikonomou, K.J. Orians, Characterization of copper-complexing ligands in seawater using immobilized copper(II)-ion affinity chromatography and electrospray ionization mass spectrometry, Mar. Chem. 83 (2003) 47-58. doi:10.1016/S03044203(03)00095-1.

[11] P. Lin, A.G. Rincon, M. Kalberer, J.Z. Yu, Elemental Composition of HULIS in the Pearl River Delta Region, China: Results Inferred from Positive and Negative Electrospray High Resolution Mass Spectrometric Data, Environ. Sci. Technol. 46 (2012) 7454-7462. doi:10.1021/es300285d.

[12] B. Helbig, R. Klöcking, P. Wutzler, Anti-Herpes Simplex Virus Type 1 Activity of Humic Acid-Like Polymers and Their O-Diphenolic Starting Compounds, Antivir. Chem. Chemother. 8 (1997) 265-273. doi:10.1177/095632029700800310.

[13] R. Klöcking, B. Helbig, G. Schötz, M. Schacke, P. Wutzler, Anti-HSV-1 activity of synthetic humic acid-like polymers derived from p-diphenolic starting compounds, Antivir. Chem. $\quad$ Chemother. $\quad 13 \quad$ (2002) 241-249. http://www.ncbi.nlm.nih.gov/pubmed/12495212.

[14] A. Bruccoleri, Positional Adaptability in the Design of Mutation-Resistant Nonnucleoside HIV-1 Reverse Transcriptase Inhibitors: A Supramolecular Perspective, AIDS Res. Hum. Retroviruses. 29 (2013) 4-12. doi:10.1089/aid.2012.0141.

[15] A. Nebbioso, A. Piccolo, Molecular characterization of dissolved organic matter (DOM): A critical review, Anal. Bioanal. Chem. 405 (2013). doi:10.1007/s00216-012-6363-2.

[16] E.C. Minor, M.M. Swenson, B.M. Mattson, A.R. Oyler, Structural characterization of dissolved organic matter: a review of current techniques for isolation and analysis, Environ. Sci. Process. Impacts. 16 (2014) 2064-2079. doi:10.1039/C4EM00062E.

[17] S. Sandron, A. Rojas, R. Wilson, N.W. Davies, P.R. Haddad, R.A. Shellie, et al., 
Chromatographic methods for the isolation, separation and characterisation of dissolved organic matter, Environ. Sci. Process. Impacts. 17 (2015) 1531-1567. doi:10.1039/c5em00223k.

[18] E.N. Capley, J.D. Tipton, A.G. Marshall, A.C. Stenson, Chromatographic Reduction of Isobaric and Isomeric Complexity of Fulvic Acids To Enable Multistage Tandem Mass Spectral Characterization, Anal. Chem. 82 (2010) 8194-8202. doi:10.1021/ac1016216.

[19] M. Hutta, R. Góra, R. Halko, M. Chalányová, Some theoretical and practical aspects in the separation of humic substances by combined liquid chromatography methods, J. Chromatogr. A. 1218 (2011) 8946-8957. doi:10.1016/j.chroma.2011.06.107.

[20] R.M.B.O. Duarte, A.C. Barros, A.C. Duarte, Resolving the chemical heterogeneity of natural organic matter: New insights from comprehensive two-dimensional liquid $\begin{array}{llllll}\text { chromatography, } & \text { J. } & \text { Chromatogr. } & \text { A. } & 1249 & \text { (2012) }\end{array}$ doi:10.1016/j.chroma.2012.06.022.

[21] Z. Liu, R.L. Sleighter, J. Zhong, P.G. Hatcher, The chemical changes of DOM from black waters to coastal marine waters by HPLC combined with ultrahigh resolution mass spectrometry, Estuar. Coast. Shelf Sci. $92 \quad$ (2011) 205-216. doi:10.1016/j.ecss.2010.12.030.

[22] A. Gaspar, M. Harir, N. Hertkorn, P. Schmitt-Kopplin, Preparative free-flow electrophoretic offline ESI-Fourier transform ion cyclotron resonance/MS analysis of Suwannee River fulvic acid, Electrophoreses. $31 \quad$ (2010) 2070-2079. doi:10.1002/elps.200900726.

[23] F.C. Wu, R.D. Evans, P.J. Dillon, Separation and Characterization of NOM by HighPerformance Liquid Chromatography and On-Line Three-Dimensional Excitation Emission Matrix Fluorescence Detection, Environ. Sci. Technol. 37 (2003) 3687-3693. doi:10.1021/es020244e.

[24] G. Plancque, B. Amekraz, V. Moulin, P. Toulhoat, C. Moulin, Molecular structure of fulvic acids by electrospray with quadrupole time-of-flight mass spectrometry, Rapid Commun. Mass Spectrom. 15 (2001) 827-835. doi:10.1002/rcm.307.

[25] B.P. Koch, K.-U. Ludwichowski, G. Kattner, T. Dittmar, M. Witt, Advanced characterization of marine dissolved organic matter by combining reversed-phase liquid chromatography and FT-ICR-MS, Mar. Chem. 111 (2008) 233-241. doi:10.1016/j.marchem.2008.05.008.

[26] A.C. Stenson, Reversed-Phase Chromatography Fractionation Tailored to Mass Spectral Characterization of Humic Substances, Environ. Sci. Technol. 42 (2008) 2060-2065. doi:10.1021/es7022412.

[27] D.B. Mawhinney, F.L. Rosario-Ortiz, S. Baik, B.J. Vanderford, S.A. Snyder, 
Characterization of fulvic acids by liquid chromatography-quadrupole time-of-flight mass $\begin{array}{lllllr}\text { spectrometry, J. } & \text { Chromatogr. } & \text { A. } & 1216 & \text { (2009) }\end{array}$ doi:10.1016/j.chroma.2008.12.068.

[28] W.-T. Li, Z.-X. Xu, A.-M. Li, W. Wu, Q. Zhou, J.-N. Wang, HPLC/HPSEC-FLD with multi-excitation/emission scan for EEM interpretation and dissolved organic matter analysis, Water Res. 47 (2013) 1246-1256. doi:10.1016/j.watres.2012.11.040.

[29] R. Góra, M. Hutta, P. Rohárik, Characterization and analysis of soil humic acids by offline combination of wide-pore octadecylsilica column reverse phase high performance liquid chromatography with narrow bore column size-exclusion chromatography and fluorescence detection, J. Chromatogr. A. 1220 (2012) 44-49. doi:10.1016/j.chroma.2011.11.044.

[30] O.A. Trubetskoj, C. Richard, G. Guyot, G. Voyard, O.E. Trubetskaya, Analysis of electrophoretic soil humic acids fractions by reversed-phase high performance liquid chromatography with on-line absorbance and fluorescence detection, J. Chromatogr. A. 1243 (2012) 62-68. doi:10.1016/j.chroma.2012.04.043.

[31] G.C. Woods, M.J. Simpson, P.J. Koerner, A. Napoli, A.J. Simpson, HILIC-NMR: Toward the Identification of Individual Molecular Components in Dissolved Organic Matter, Environ. Sci. Technol. 45 (2011) 3880-3886. doi:10.1021/es103425s.

[32] G.C. Woods, M.J. Simpson, A.J. Simpson, Oxidized sterols as a significant component of dissolved organic matter: Evidence from 2D HPLC in combination with 2D and 3D NMR spectroscopy, Water Res. 46 (2012) 3398-3408. doi:10.1016/j.watres.2012.03.040.

[33] B.D. Harris, T.A. Brown, J.L. McGehee, D. Houserova, B.A. Jackson, B.C. Buchel, et al., Characterization of Disinfection By-Products from Chromatographically Isolated NOM through High-Resolution Mass Spectrometry, Environ. Sci. Technol. 49 (2015) 1423914248. doi:10.1021/acs.est.5b03466.

[34] A.C. Stenson, W.M. Landing, A.G. Marshall, W.T. Cooper, Ionization and Fragmentation of Humic Substances in Electrospray Ionization Fourier Transform-Ion Cyclotron Resonance Mass Spectrometry, Anal. Chem. 74 (2002) 4397-4409. doi:10.1021/ac020019f.

[35] E.B. Kujawinski, P.G. Hatcher, M.A. Freitas, High-Resolution Fourier Transform Ion Cyclotron Resonance Mass Spectrometry of Humic and Fulvic Acids: Improvements and Comparisons, Anal. Chem. 74 (2002) 413-419. doi:10.1021/ac0108313.

[36] E.B. Kujawinski, M.A. Freitas, X. Zang, P.G. Hatcher, K.B. Green-Church, R.B. Jones, The application of electrospray ionization mass spectrometry (ESI MS) to the structural characterization of natural organic matter, Org. Geochem. 33 (2002) 171-180. doi:10.1016/S0146-6380(01)00149-8. 
[37] T. Reemtsma, A. These, A. Springer, M. Linscheid, Fulvic Acids as Transition State of Organic Matter: Indications from High Resolution Mass Spectrometry, Environ. Sci. Technol. 40 (2006) 5839-5845. doi:10.1021/es060318c.

[38] A.C. Stenson, A.G. Marshall, W.T. Cooper, Exact Masses and Chemical Formulas of Individual Suwannee River Fulvic Acids from Ultrahigh Resolution Electrospray Ionization Fourier Transform Ion Cyclotron Resonance Mass Spectra, Anal. Chem. 75 (2003) 1275-1284. doi:10.1021/ac026106p.

[39] E.B. Kujawinski, R. Del Vecchio, N. V. Blough, G.C. Klein, A.G. Marshall, Probing molecular-level transformations of dissolved organic matter: insights on photochemical degradation and protozoan modification of DOM from electrospray ionization Fourier transform ion cyclotron resonance mass spectrometry, Mar. Chem. 92 (2004) 23-37. doi:10.1016/j.marchem.2004.06.038.

[40] L.B. Tremblay, T. Dittmar, A.G. Marshall, W.J. Cooper, W.T. Cooper, Molecular characterization of dissolved organic matter in a North Brazilian mangrove porewater and mangrove-fringed estuaries by ultrahigh resolution Fourier Transform-Ion Cyclotron Resonance mass spectrometry and excitation/emission spectroscopy, Mar. Chem. 105 (2007) 15-29. doi:10.1016/j.marchem.2006.12.015.

[41] K. Mopper, A. Stubbins, J.D. Ritchie, H.M. Bialk, P.G. Hatcher, Advanced Instrumental Approaches for Characterization of Marine Dissolved Organic Matter: Extraction Techniques, Mass Spectrometry, and Nuclear Magnetic Resonance Spectroscopy, Chem. Rev. 107 (2007) 419-442. doi:10.1021/cr050359b.

[42] A.C. Stenson, B.M. Ruddy, B.J. Bythell, Ion molecule reaction H/D exchange as a probe for isomeric fractionation in chromatographically separated natural organic matter, Int. J. Mass Spectrom. 360 (2014) 45-53. doi:10.1016/j.ijms.2013.12.026.

[43] P. Dugo, F. Cacciola, T. Kumm, G. Dugo, L. Mondello, Comprehensive multidimensional liquid chromatography: Theory and applications, J. Chromatogr. A. 1184 (2008) 353-368. doi:10.1016/j.chroma.2007.06.074.

[44] I. François, K. Sandra, P. Sandra, Comprehensive liquid chromatography: Fundamental aspects and practical considerations-A review, Anal. Chim. Acta. 641 (2009) 14-31. doi:10.1016/j.aca.2009.03.041.

[45] H. Malerod, E. Lundanes, T. Greibrokk, Recent advances in on-line multidimensional liquid chromatography, Anal. Methods. 2 (2010) 110-122. doi:10.1039/B9AY00194H.

[46] P. Donato, F. Cacciola, P.Q. Tranchida, P. Dugo, L. Mondello, Mass spectrometry detection in comprehensive liquid chromatography: Basic concepts, instrumental aspects, applications and trends, Mass Spectrom. Rev. 31 (2012) 523-559. doi:10.1002/mas.20353. 
[47] A.G. Marshall, T.C.L. Wang, T.L. Ricca, Tailored excitation for Fourier transform ion cyclotron mass spectrometry, J. Am. Chem. Soc. 107 (1985) 7893-7897. doi:10.1021/ja00312a015.

[48] S. Guan, A.G. Marshall, Stored waveform inverse Fourier transform (SWIFT) ion excitation in trapped-ion mass spectometry: Theory and applications, Int. J. Mass Spectrom. Ion Process. 157 (1996) 5-37. doi:10.1016/S0168-1176(96)04461-8.

[49] K. Hakansson, M.J. Chalmers, J.P. Quinn, M.A. McFarland, C.L. Hendrickson, A.G.

[50] N.K. Kaiser, J.P. Quinn, G.T. Blakney, C.L. Hendrickson, A.G. Marshall, A novel 9.4 tesla FTICR mass spectrometer with improved sensitivity, mass resolution, and mass range, J. Am. Soc. Mass Spectrom. 22 (2011) 1343-1351. doi:10.1007/s13361-011-01419.

[51] B.J. Bythell, C.L. Hendrickson, A.G. Marshall, Relative stability of peptide sequence ions generated by tandem mass spectrometry, J. Am. Soc. Mass Spectrom. 23 (2012) 644-654. doi:10.1007/s13361-012-0357-3.

[52] N. Hertkorn, C. Ruecker, M. Meringer, R. Gugisch, M. Frommberger, E.M. Perdue, et al., High-precision frequency measurements: indispensable tools at the core of the molecularlevel analysis of complex systems, Anal. Bioanal. Chem. 389 (2007) 1311-1327. doi:10.1007/s00216-007-1577-4.

[53] H. Yabut, M. Fukushima, M. Kawasaki, F. Tanaka, T. Kobayashi, K. Tatsumi, Multiple polar components in poorly-humified humic acids stabilizing free radicals : Carboxyl and nitrogen-containing carbons, Org. Geochem. 39 (2008) 1319-1335. http://cat.inist.fr/?aModele=afficheN\&cpsidt=20659607 (accessed March 6, 2016).

[54] R. Vessecchi, A.E. M. Crotti, T. Guaratini, P. Colepicolo, S.E. Galembeck, N.P. Lopes, Radical Ion Generation Processes of Organic Compounds in Electrospray Ionization Mass Spectrometry, Mini. Rev. Org. Chem. 4 (2007) 75-87.

[55] A.G. Marshall, C.L. Hendrickson, G.S. Jackson, Fourier transform ion cyclotron resonance mass spectrometry: a primer, Mass Spectrom. Rev. 17 (1998) 1-35. doi:10.1002/(SICI)1098-2787(1998)17:1<1::AID-MAS1>3.0.CO;2-K.

[56] M. Witt, J. Fuchser, B.P. Koch, Fragmentation Studies of Fulvic Acids Using Collision Induced Dissociation Fourier Transform Ion Cyclotron Resonance Mass Spectrometry, Anal. Chem. 81 (2009) 2688-2694. doi:10.1021/ac802624s.

[57] William C. Hockaday, Jeremiah M. Purcell, Alan G. Marshall, Patrick G. Hatcher, Electrospray and photoionization mass spectrometry for the characterization of organic 
matter in natural waters: a qualitative assessment, Limnol. Oceanogr. Methods. 7 (2009) 81-95. 
Figure 1. Correlation of retention times of humic-like standards between both second dimension methods and the first dimension method. Correlation between the column 1 and the second dimension method optimized for early eluting material (column 2 method) is low indicating near orthogonality: $\mathrm{r}^{2}=0.3762$. The correlation of retention times for standards through column 1 and the second dimension method optimized for late eluting material (column 2' method) is even closer to orthogonality with an $r^{2}$ value of 0.0003 .

Figure 2. SRFA chromatograms. A\&B. SRFA through column 1; in A FRC05 is highlighted in blue; in, B. FRC53 is highlighted. C. UV-Vis chromatogram of FRC05 re-injected on column 1. D. ESI-LCMS extracted ion chromatograms of several additional column 1 fractions (FRC06, 10, 15, 20, 25, 29, 36, 39, and 49) re-injected onto column 1. Dashed black lines represent features induced from drastic changes in the mobile phase gradient; equivalent features are observed in all column 1 chromatograms (A-E). E. UV-Vis chromatogram of FRC53 re-injected on column 1. F. UV-Vis (he column 2, G. UV-Vis chromatogram of FRC53 through column 2'. H. Extractedion chromatogram of FRC05_45 re-injected on column 2, I. Extracted-ion chromatogram of the combination of fractions FRC53_27 and FRC53_29 re-injected onto column 2'. Low sample size in the second dimension necessitated a switch from UV-Vis to MS detection (green traces for MS; blue traces for UV-vis).

Figure 3: Oxygen class distributions for second dimension (column 2 and 2') fractions: The $x$-axis is the number of $O$ atoms in each formula from $\mathrm{O}_{1}-\mathrm{O}_{19}$ A. Second dimension sub-fractions of FRC05 (fractions: FRC05_17, FRC05_23, FRC05_45 and FRC05_67). B. Second dimension sub-fractions of FRC53 (fractions: FRC53_11, FRC53_28, and FRC53_67). Retention times of fractions are listed in Table 1.

Figure 4: Van Krevelen plots of second dimension subfractions of FRC05 and FRC53. A. FRC05_17 B. FRC05_23 C. FRC05_67 D. FRC53_11 E. FRC53_28 F. FRC53_67. Subfractions from FRC05 are represented in blue whereas those from FRC53 are represented in red. Any radicals formed are represented in a darker color. Boxes represent: i. Lipid-like region, ii. Carbohydrate-like region, and iii. CAS-like region. Regions adapted from Hockaday et al.[57]

Figure 5: Abundance distribution of even electron ions and radicals for the second dimension subfractions of FRC53. A.

Figure 6: Van Krevelen Plot of first dimension Fractions. Early eluting NOM is shown in blue, middle in black, and late in red. Boxes represent regions characteristic of Lipids, Proteins, Carbohydrates, Lignin, and Condensed Aromatic Structures. Dashed lines represent overlapping regions. Regions adapted from Hockaday et al.[57]

Figure 7: Correlation between SciFinder Hits and Fragment mass. For each fragment formula, the number of compounds with the identical formula were counted in the SciFinder database. The base 10 logarithm of that number is plotted against the fragment mass. Colors differentiate the degree of oxidation of the fragments. Dark Blue: Low H/O ratio, represents the most oxidized (typically early-eluting). Dark Red: High H/O ratio, represents the least oxidized (typically late-eluting). Note, because the log of zero is undefined, both no hits and 1 hit were assigned a y-value of zero here (plotted on the xaxis).

Figure 8: Best and worst obtainable results for fragmentation patterns of isomers - FRC05 through the column 2 LC MS/MS method and FRC53 through the column 2' LCMS/MS method. Losses of $\mathrm{H}_{2} \mathrm{O}(\mathrm{m} / \mathrm{z} 18)$ and $\mathrm{CO}_{2}(\mathrm{~m} / \mathrm{z} 44)$ are plotted as a ratio of fragment to the 'parent peak' normalized to the largest ratio observed in each chromatogram for $\mathbf{A}$. FRC05 at m/z 371 (best), B. FRC05 at m/z 297 (worst), C. FRC53 at m/z 383 (best), and D. FRC53 at m/z 453 (worst).

Figure 9. Ratio of fragment to parent ion for standards mixed with the mobile phase stream for column 2 and 2' method.. A. Column 2 method, citric acid (standard 17) B. Column 2' method, maleic acid (standard 12 ) C. Column 2 method, naproxen (standard 23) D. Column 2' method, naproxen. Standard structures are shown within their respective plots. Losses of $\mathrm{H}_{2} \mathrm{O}(\mathrm{m} / \mathrm{z} 18)$ and $\mathrm{CO}_{2}(\mathrm{~m} / \mathrm{z} 44)$ plotted as a ratio of fragment to the 'parent peak' normalized to the largest ratio observed in each chromatogram. 


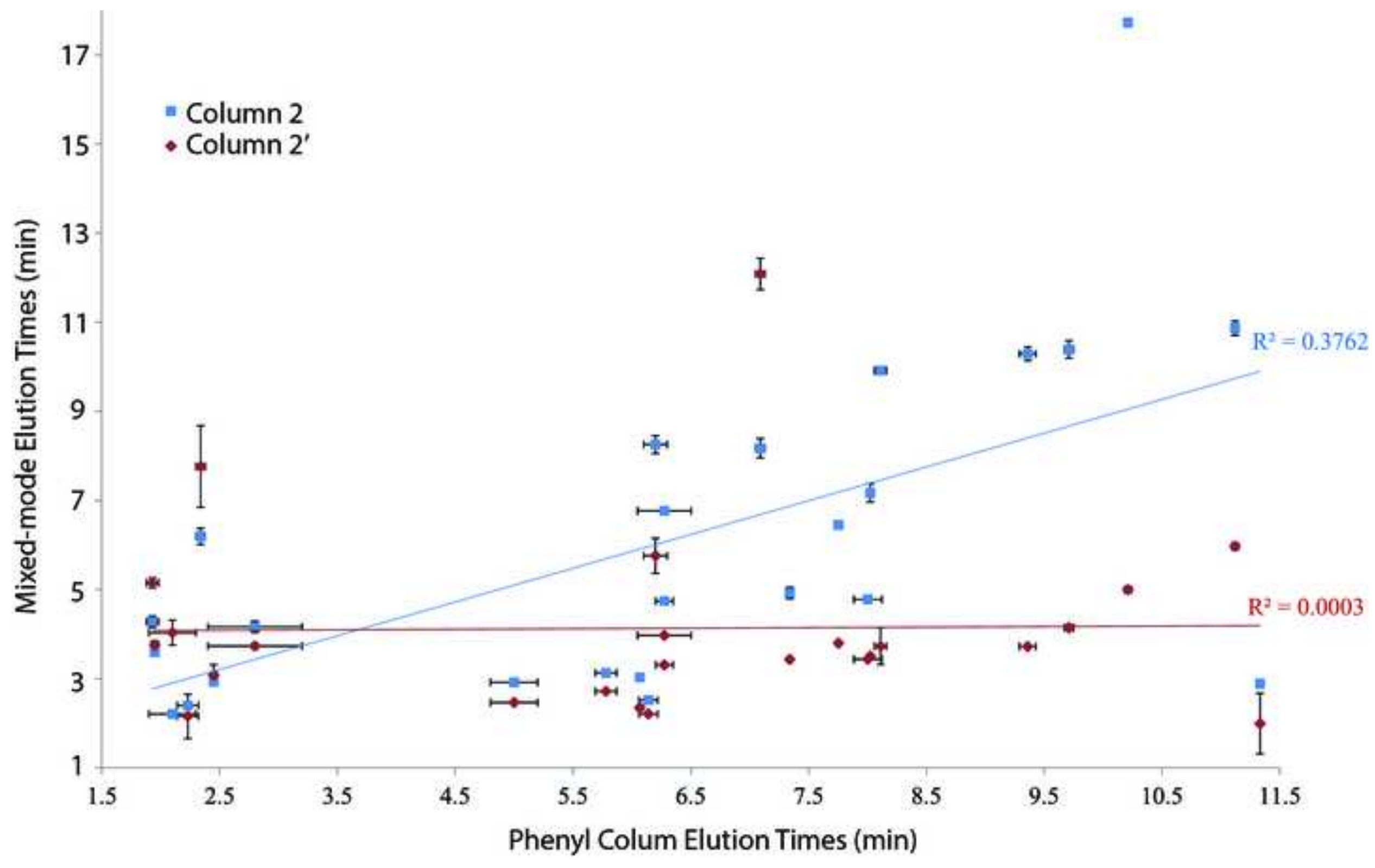


Figure 2
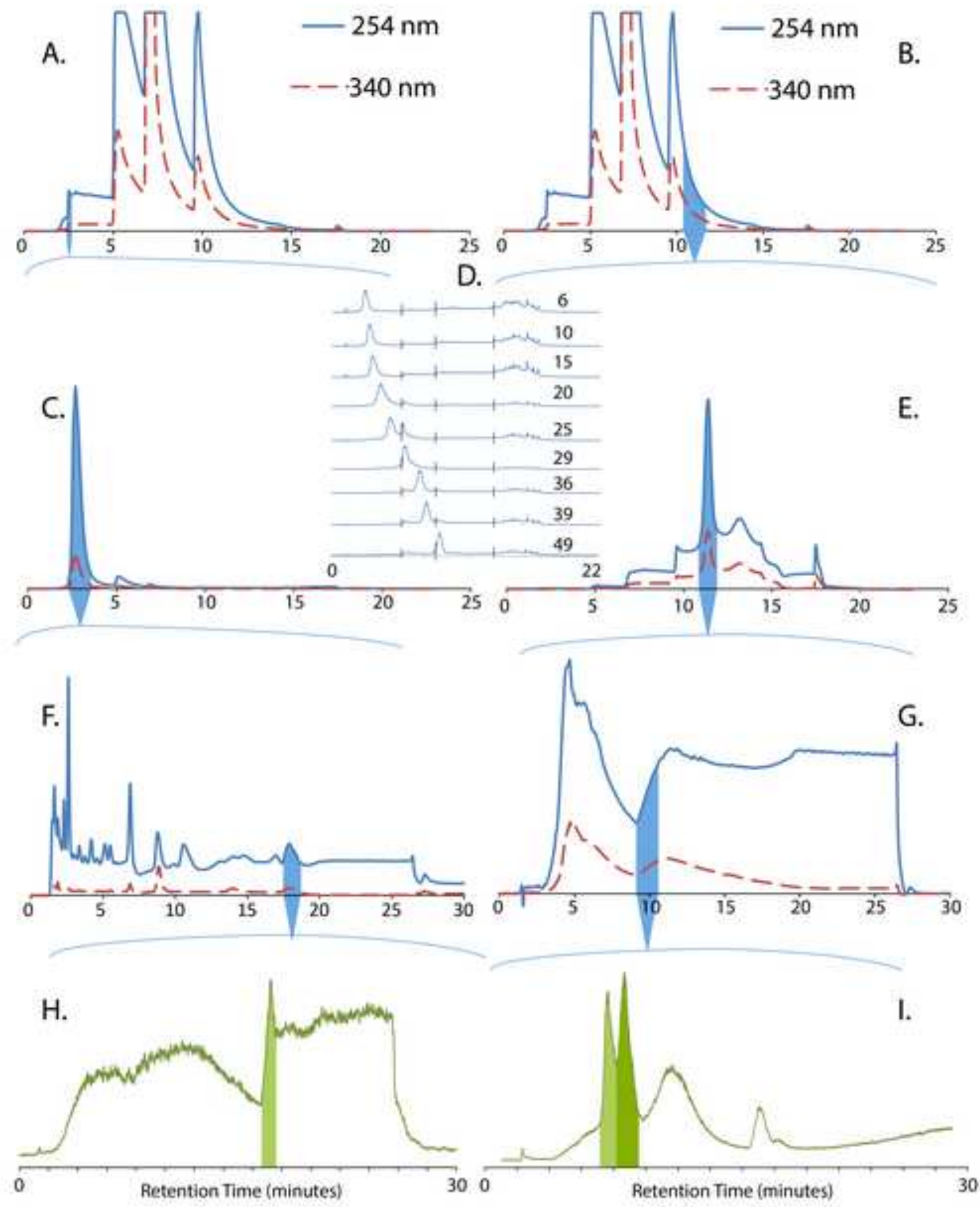
Figure 3

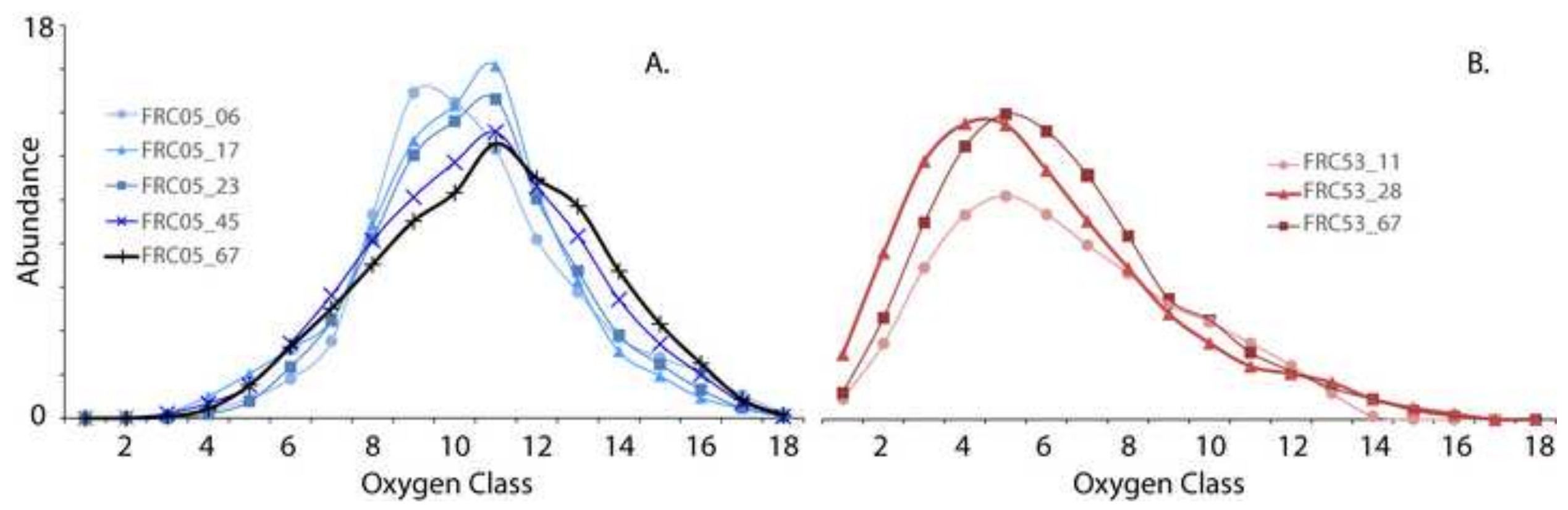


Figure 4
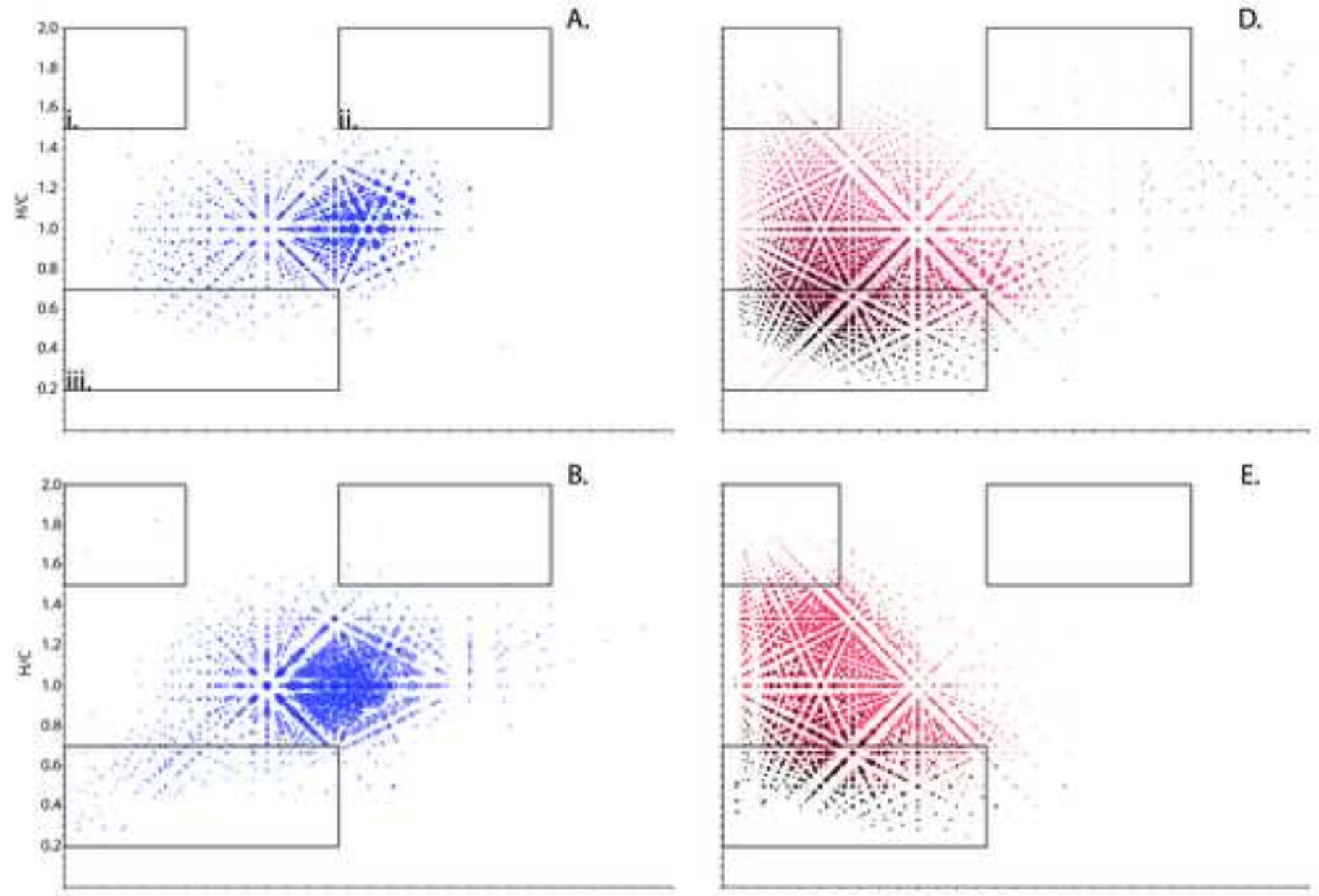

E.
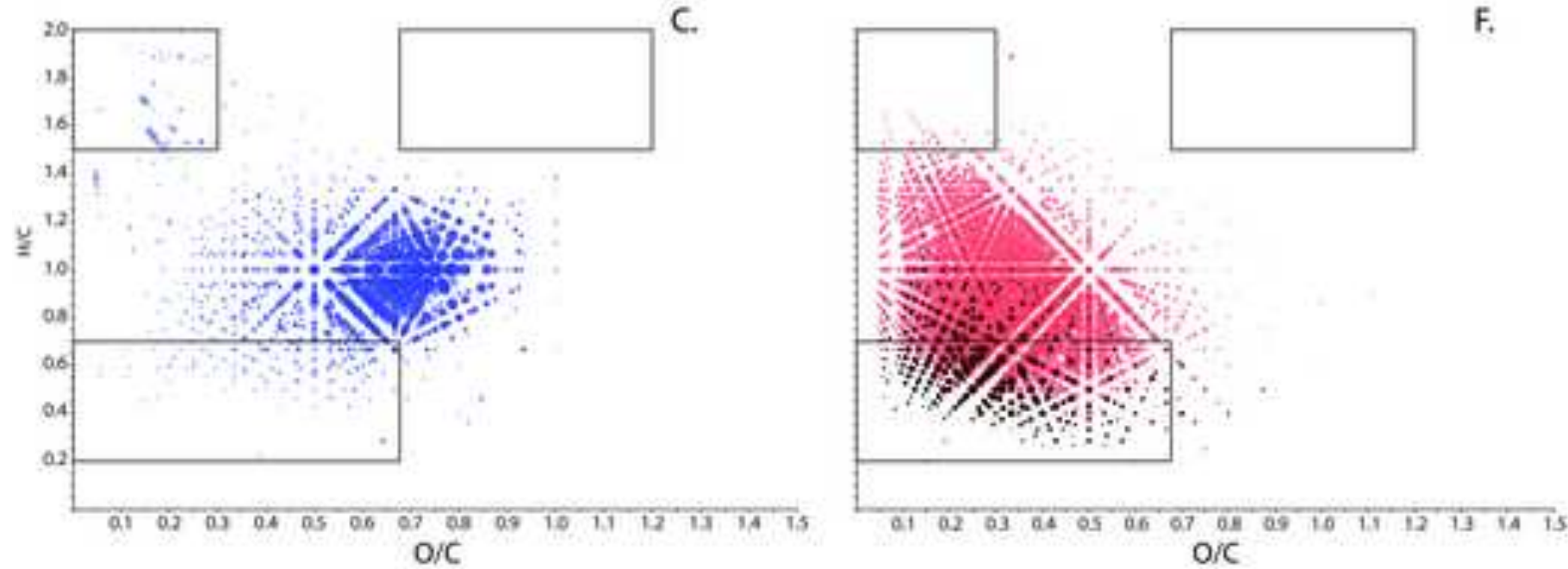


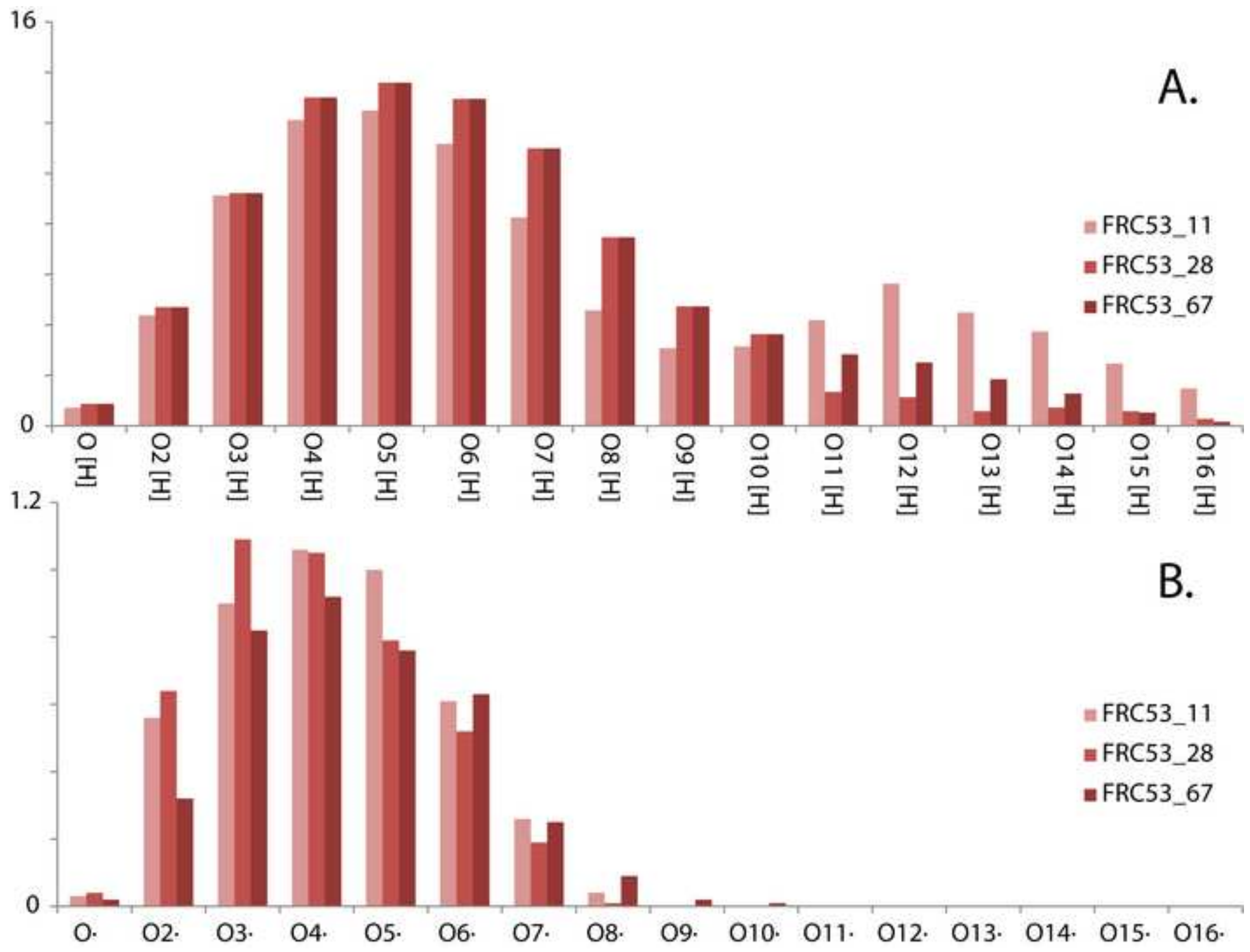




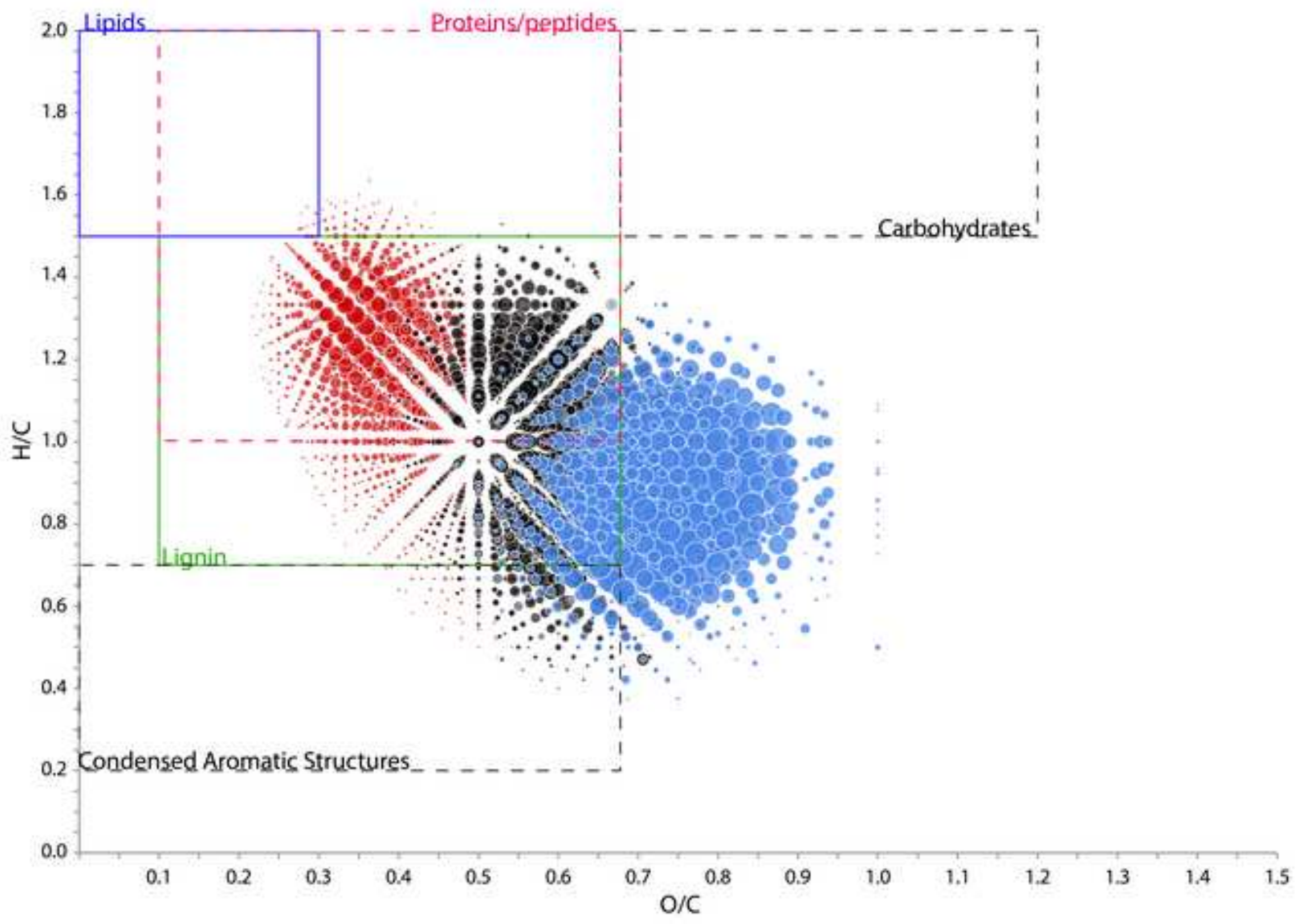




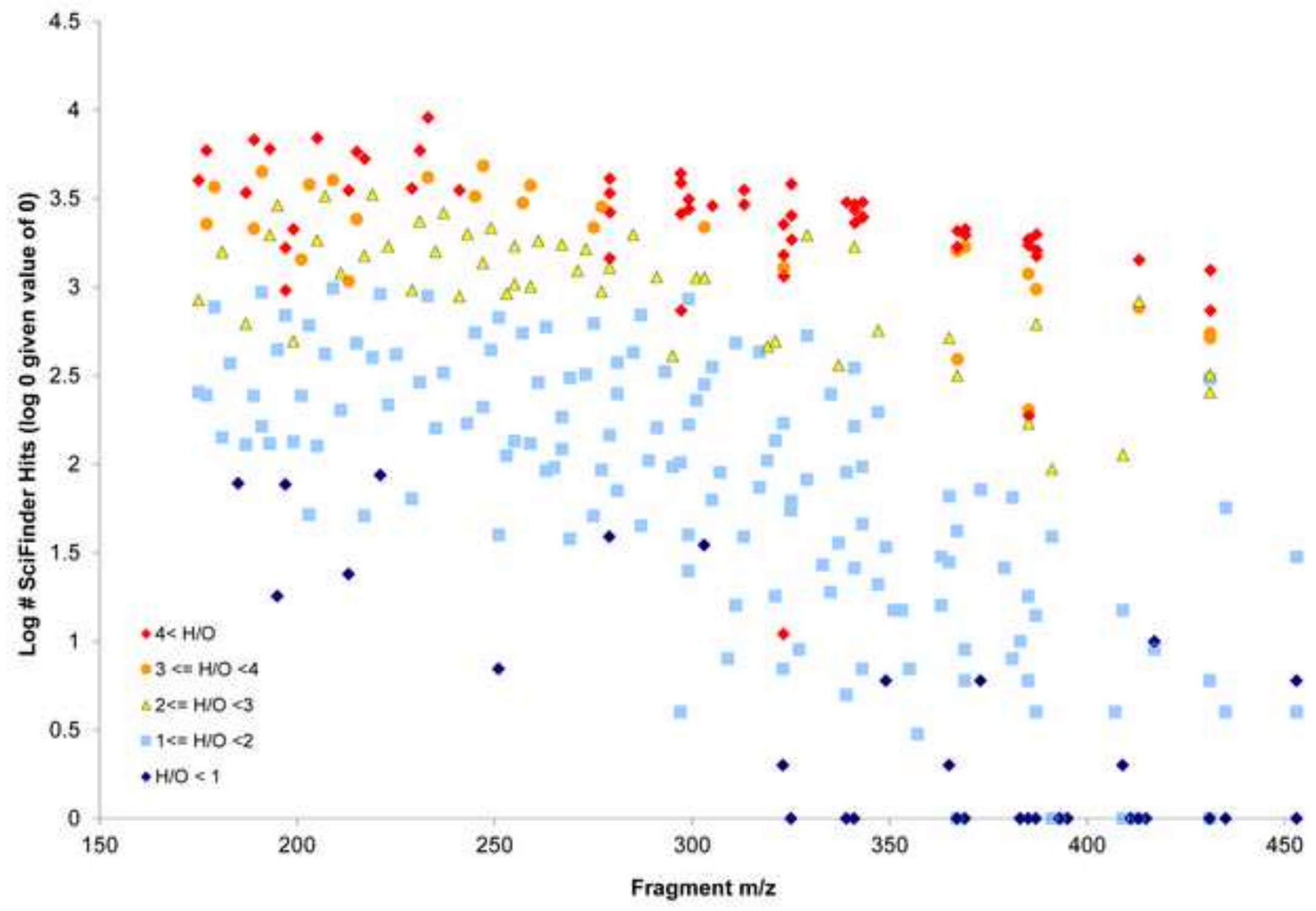



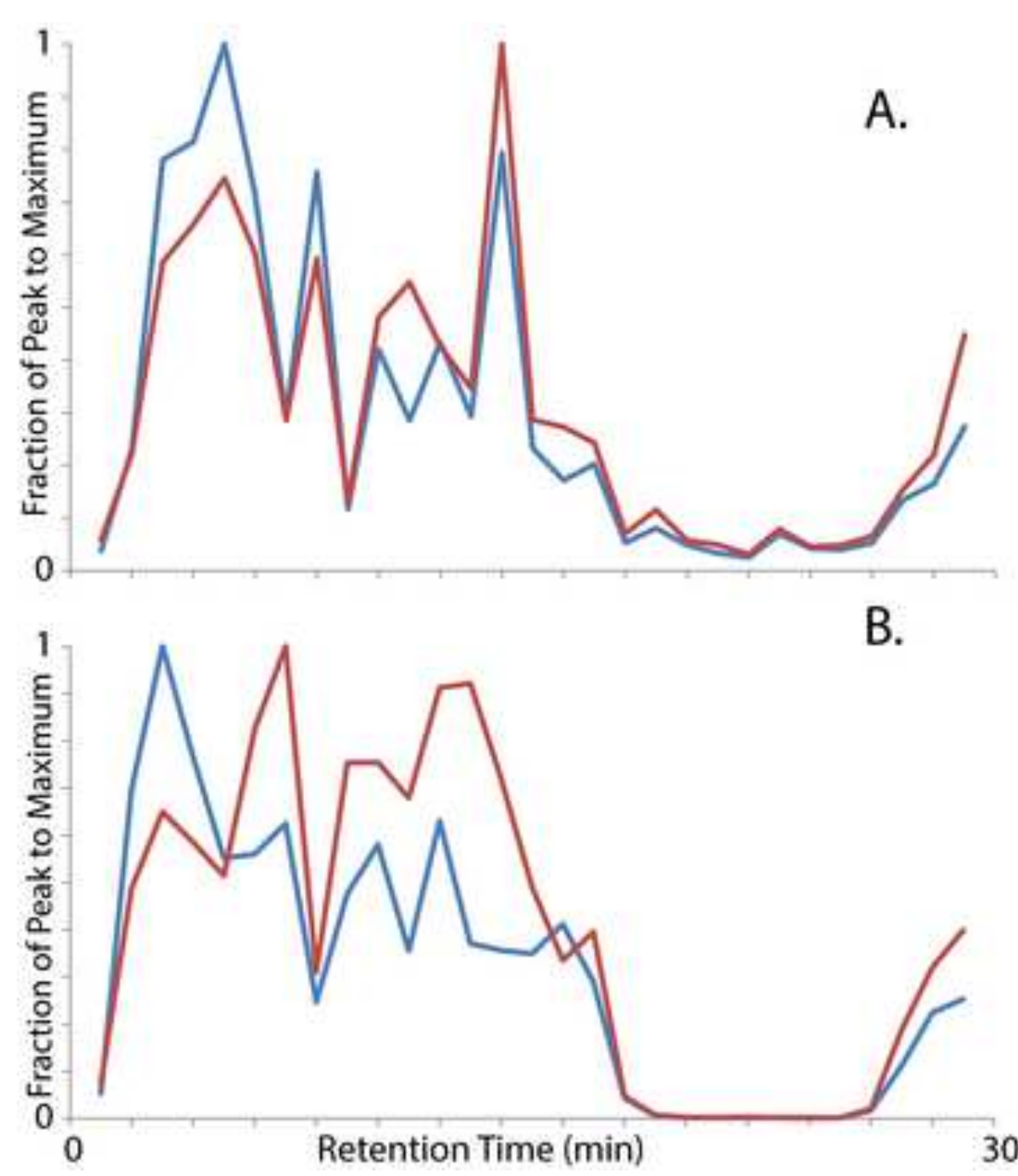

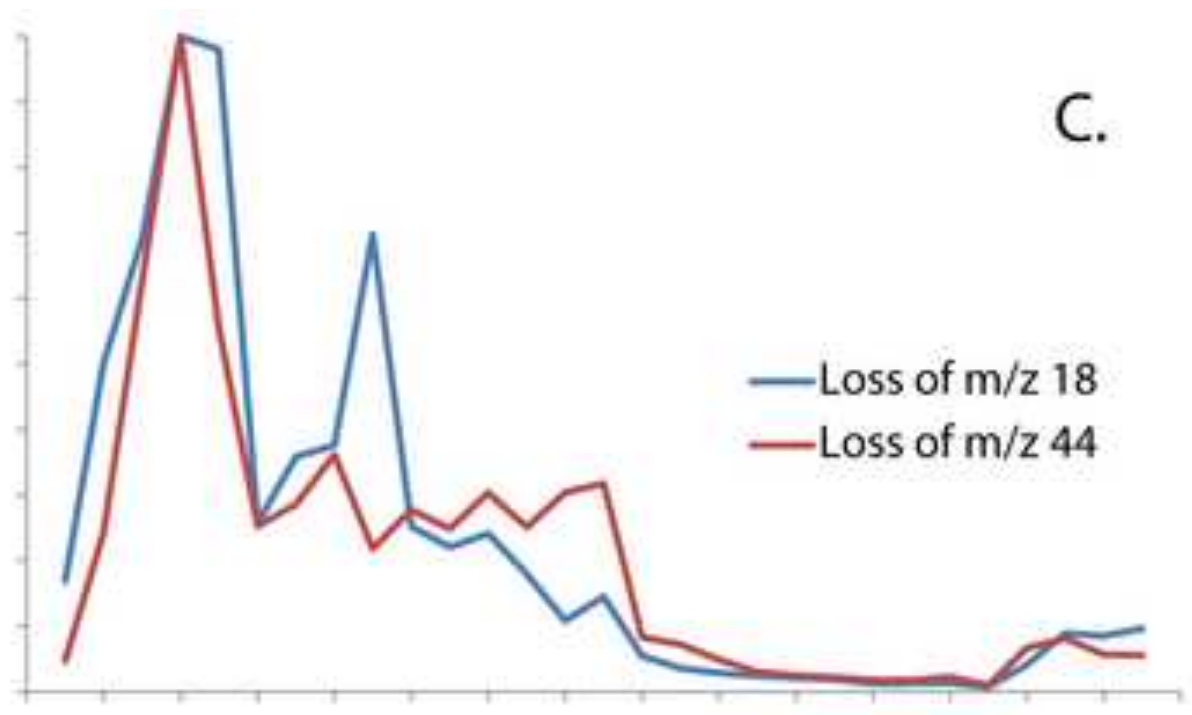

D.

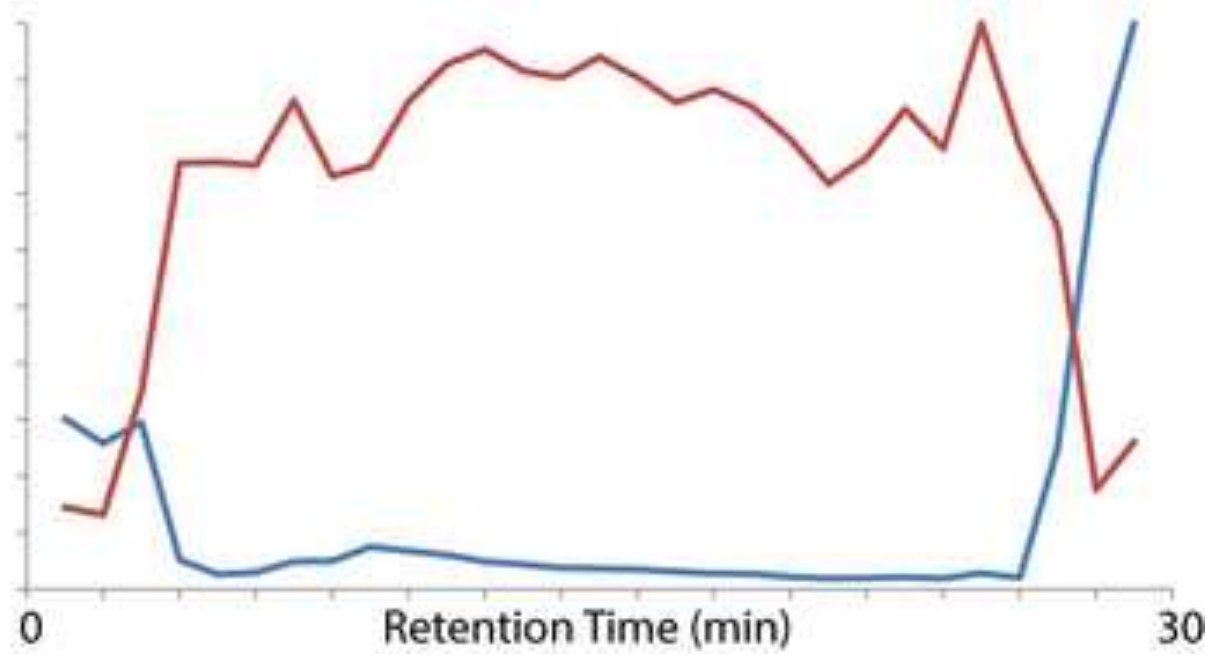


A.
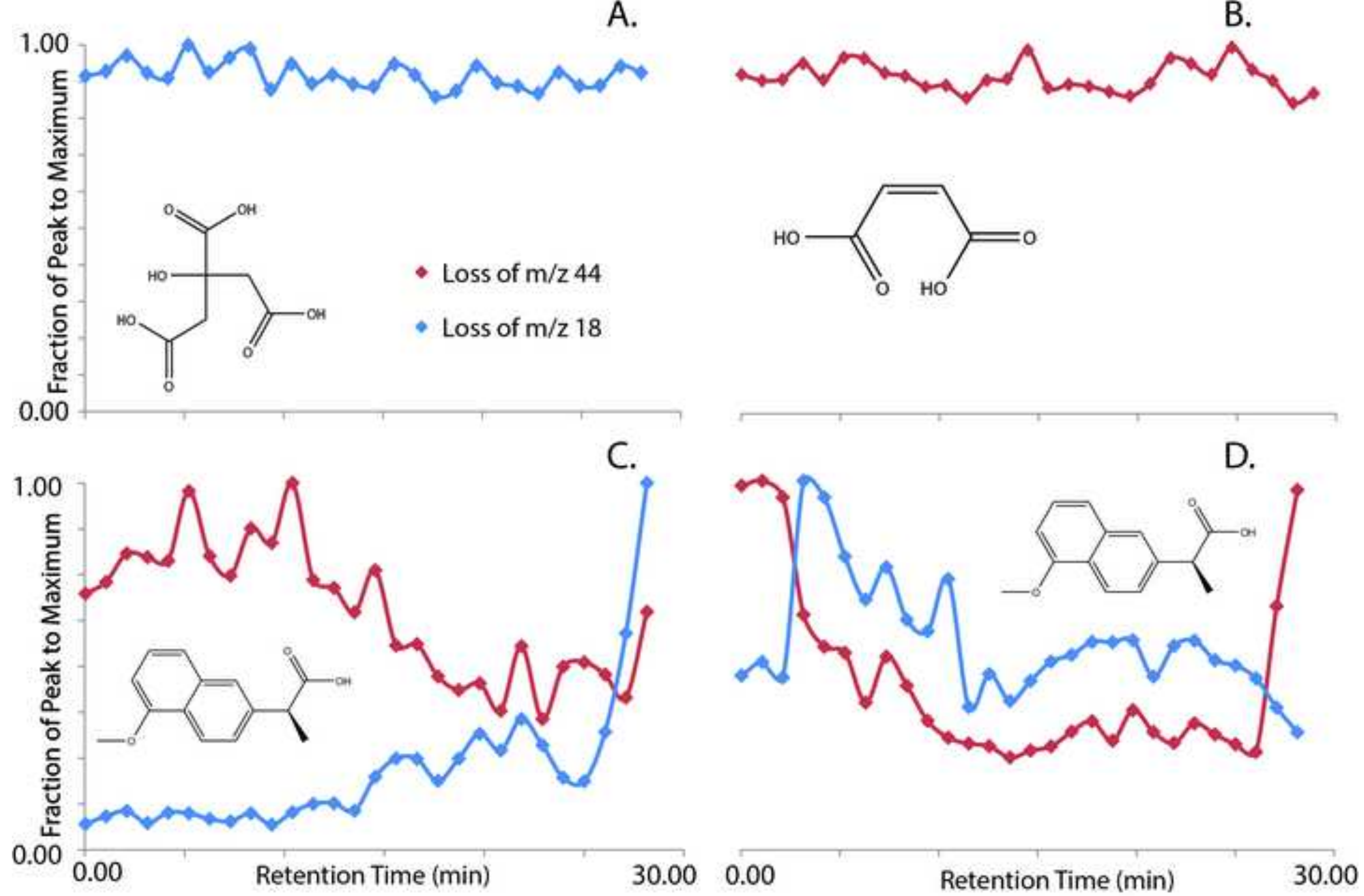


\begin{tabular}{|c|c|c|}
\hline Sample I.D. & $\begin{array}{c}\text { 1D Retention } \\
\text { Time (minutes) }\end{array}$ & $\begin{array}{c}\text { 2D Retention } \\
\text { Time (minutes) }\end{array}$ \\
\hline FRC05_17 & 3 & 7 \\
\hline FRC05_23 & 3 & 8 \\
\hline FRC05_45 & 3 & 18 \\
\hline FRC05_67 & 3 & 26.5 \\
\hline FRC53_11 & 11.5 & 4.5 \\
\hline FRC53_28 & 11.5 & 11 \\
\hline FRC53_67 & 11.5 & 26.5 \\
\hline
\end{tabular}

Table 1. Retention times for 1D and 2D SRFA fractions. 